\title{
Deep Inelastic Pion Electroproduction at Threshold
}

\author{
V. M. Braun ${ }^{1}$, D. Yu. Ivanov ${ }^{2}$, A. Lenz ${ }^{1}$ and A. Peters ${ }^{1}$ \\ 1 Institut für Theoretische Physik, Universität Regensburg, D-93040 Regensburg, Germany \\ 2 Sobolev Institute of Mathematics, 630090 Novosibirsk, Russia
}

(Dated: November 1, 2018)

\begin{abstract}
We consider the cross section of the deep inelastic pion electroproduction on a proton target at threshold for $Q^{2}$ in the region $5-10 \mathrm{GeV}^{2}$. The corresponding amplitudes are described in terms of two form factors which we calculate using light cone sum rules (LCSR) to leading order in QCD and including higher twist corrections. Our results suggest a considerable change from a small $Q^{2}$ region that can be treated in the soft pion limit using current algebra. In particular, we obtain a $\pi^{0}$ to $\pi^{+}$producton ratio of order $1 / 3$ and significant nucleon helicity-flip contributions.
\end{abstract}

PACS numbers: 12.38.-t, 14.20.Dh; 13.40.Gp

\section{INTRODUCTION}

In recent years there has been increasing attention to hard exclusive processes involving emission of soft pions in the final state. One reason is that such processes often provide the main background to the simpler reactions that one wants to study, and one would like to estimate the background as precise as possible. The main motivation is, however, different. It turns out that hard production of soft pions is interesting in its own right and can provide one with new insights in the hadron (nucleon) structure. The novel physical feature is in this case the presence of three disparate scales: the large momentum transfer $Q$, the QCD scale $\Lambda=\Lambda_{\mathrm{QCD}}$, and the pion mass $m_{\pi}$ which goes to zero in the chiral limit, so that $Q \gg \Lambda_{\mathrm{QCD}} \gg m_{\pi}$. Moreover, the limits $m_{\pi} \rightarrow 0$ and $Q \rightarrow \infty$ do not commute. If, parametrically, $Q^{2} \ll \Lambda^{3} / m_{\pi}$ then the standard approach based on chiral symmetry and current algebra is applicable. In this way the celebrated soft pion theorems arise 1]: the amplitudes with pion emission are calculated in terms of the amplitudes without pions induced by a chirally-rotated current, and pion emission from the external hadron lines. There exists vast literature on this topic, see e.g. 2, 3, 4], extending the original Kroll-Ruderman prediction to include corrections in the pion mass and/or the pion (photon) momentum.

In the opposite limit $Q^{2} \gg \Lambda^{3} / m_{\pi}$ these techniques do not apply, and the accepted general philosophy is to use QCD factorization to separate contributions of large momenta of the order of the hard scale $Q$ in perturbatively calculable "coefficient functions" in front of nonperturbative matrix elements that contain contributions of smaller scales. The methods of current algebra (or chiral perturbation theory) can then be used to simplify the matrix elements rather than the full physical amplitude in question. The present study is fuelled by the ob- servation made in Ref. [5] that in the asymptotic $Q \rightarrow \infty$ limit the pion electroproduction can be described in the framework of the usual pQCD factorization formalism for exclusive processes [ $[6,7,6]$ and involve an overlap integral of chirally rotated nucleon light-cone distribution amplitudes (DAs). The particular combination of the DAs involved in the pion electroproduction turns out to be different from that in the electromagnetic (or weak) elastic nucleon form factors, so that different components of DAs potentially could be separated from the comparison. This is interesting, since nucleon light-cone DAs remain to be poorly known. Similar ideas have been applied in a somewhat different context in Refs. 9, 10].

The essential requirement for the applicability of the pQCD factorization is a high virtuality of the exchanged gluons and also of the quarks inside the short distance subprocess. The main problem is a numerical suppression of each hard gluon exchange by the $\alpha_{s} / \pi$ factor which is a standard perturbation theory penalty for each extra loop. If, say, $\alpha_{s} / \pi \sim 0.1$, the pQCD contribution to baryon form factors is suppressed by a factor of 100 compared to the purely soft term. As the result, the onset of the perturbative regime is postponed to very large momentum transfers since the factorizable pQCD contribution has to win over (nonfactorizable) nonperturbative effects that are suppressed by extra powers of $1 / Q^{2}$ but do not involve small coefficients. There is a growing consensus that such "soft" contributions play the dominant role at present energies. Indeed, it is known for a long time that the use of QCD-motivated models for the wave functions allows one to obtain, without much effort, soft contributions comparable in size to experimentally observed values (see, e.g. [11, 12, 13]). A current fashion [14, 15] is to use the concept of generalized parton distributions (GPDs) to describe/parametrize soft contributions in various exclusive reactions, see 
[16, 17, 18] for recent reviews, and the models of GPDs usually are chosen such that the experimental data on form factors are described by the soft contributions alone, cf. Refs. [19, 20, 21]. The soft pion electroproduction was considered in this framework in [22, 23]. A subtle point for these semi-phenomenological approaches is to avoid double counting of hard rescattering contributions "hidden" in the model-dependent hadron wave functions or GPD parametrizations.

Another approach to the calculation of form factors for moderately large $Q^{2}$ is based on the lightcone sum rules (LCSR) 24, 25]. This technique is attractive because in LCSRs "soft" contributions to the form factors are calculated in terms of the same DAs that enter the pQCD calculation and there is no double counting. Thus, the LCSRs provide one with the most direct relation of the hadron form factors and distribution amplitudes that is available at present, with no other nonperturbative parameters.

In this paper, we suggest to use light-cone sum rules to calculate the amplitudes of pion electroproduction. The basic object of the LCSR approach is the correlation function

$$
\int d x e^{-i q x}\langle N(P) \pi(k)|T\{j(x) \eta(0)\}| 0\rangle
$$

in which $j$ represents the electromagnetic probe and $\eta$ is a suitable operator with nucleon quantum numbers. The final state nucleon and the pion are explicitly represented by the state vector $\langle N(P) \pi(k)|$, see a schematic representation in Fig. 11 When

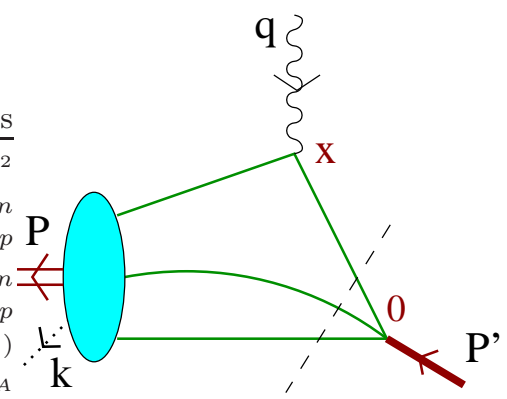

FIG. 1: Schematic structure of the light-cone sum rule for pion electroproduction

both the momentum transfer $Q^{2}$ and the momentum $\left(P^{\prime}\right)^{2}=(P-q+k)^{2}$ flowing in the $\eta$ vertex are large and negative, the asymptotics of the correlation function is governed by the light-cone kinematics $x^{2} \rightarrow 0$ and can be studied using the operator product expansion (OPE) $T\{j(x) \eta(0)\} \sim \sum C_{i}(x) \mathcal{O}_{i}(0)$ on the light-cone $x^{2}=0$. The $x^{2}$-singularity of a particular perturbatively calculable short-distance factor $C_{i}(x)$ is determined by the twist of the relevant composite operator $\mathcal{O}_{i}$, whose matrix element $\left\langle N(P) \pi(k)\left|\mathcal{O}_{i}(0)\right| 0\right\rangle$ is given by an appropriate moment of the (complex conjugated) pion-nucleon generalized DA. Using current algebra and chiral symmetry these matrix element can be reduced to the usual nucleon DAs. Next, one can represent the answer in form of the dispersion integral in $\left(P^{\prime}\right)^{2}$ and define the nucleon contribution by the cutoff in the invariant mass of the three-quark system, the socalled interval of duality $s_{0}$ (or continuum threshold). The main role of the interval of duality is that it does not allow large momenta $\left|k^{2}\right|>s_{0}$ to flow through the $\eta$-vertex; to the lowest order $O\left(\alpha_{s}^{0}\right)$ one obtains a purely soft contribution to the form factor as a sum of terms ordered by twist of the relevant operators and hence including both the leadingand the higher-twist nucleon DAs. Note that, in difference to the hard mechanism, the contribution of higher-twist DAs is only suppressed by powers of $\left|\left(P^{\prime}\right)^{2}\right| \sim 1-2 \mathrm{GeV}^{2}$ (which is translated to the suppression by powers of the Borel parameter after applying the usual QCD sum rule machinery), but not by powers of $Q^{2}$. This feature is in agreement with the common wisdom that soft contributions are not constrained to small transverse separations.

The LCSR expansion also contains terms generating the asymptotic pQCD contributions. They appear at proper order in $\alpha_{s}$, i.e., in the $O\left(\alpha_{s}\right)$ term for the pion form factor, at the $\alpha_{s}^{2}$ order for the nucleon form factors, etc. In the pion case, it was explicitly demonstrated [26, 27] that the contribution of hard rescattering is correctly reproduced in the LCSR approach as a part of the $O\left(\alpha_{s}\right)$ correction. It should be noted that the diagrams of LCSR that contain the "hard" pQCD contributions also possess "soft" parts, i.e., one should perform a separation of "hard" and "soft" terms inside each diagram. As a result, the distinction between "hard" and "soft" contributions appears to be scale- and scheme-dependent [26]. During the last years there have been numerous applications of LCSRs to mesons, see [28, 29] for a review. Following the work 30] nucleon electromagnetic form factors were further considered in this framework in Refs. [31, 32, 33, 34] and the weak decays $\Lambda_{b} \rightarrow p \ell \nu_{\ell}, \Lambda_{c} \rightarrow \Lambda \ell \nu_{\ell}$ etc. in [35, 36, 37]. A generalization to the $N \gamma \Delta$ transition form factor was worked out in [38].

The presentation is organized as follows. Section 2 is mainly introductory. We introduce here the necessary notations, explain the kinematics and define the two independent invariant amplitudes (generalized form factors) that are relevant for the pion electroproduction at threshold. The main nonperturbative input in our calculations is provided by the general- 
ized pion-nucleon distribution amplitudes which are calculated using current algebra in Section 3. The light-cone sum rules are constructed in Section 4. The numerical analysis is done in Section 5 which contains our main results. Finally, Section 6 contains a short summary and conclusions.

\section{KINEMATICS AND NOTATIONS}

In this paper we consider pion electroproduction from a proton target

$$
\begin{aligned}
& e\left(l^{\prime}\right)+p\left(P^{\prime}\right) \rightarrow e(l)+\pi^{+}(k)+n(P), \\
& e\left(l^{\prime}\right)+p\left(P^{\prime}\right) \rightarrow e(l)+\pi^{0}(k)+p(P) .
\end{aligned}
$$

Basic kinematic variables are

$$
\begin{aligned}
& q=l^{\prime}-l, \quad s=\left(l^{\prime}+P^{\prime}\right)^{2}, \quad W^{2}=(k+P)^{2}, \\
& q^{2}=-Q^{2}, \quad P^{\prime 2}=P^{2}=m_{N}^{2}, \quad k^{2}=m_{\pi}^{2} \\
& y=\frac{P^{\prime} \cdot q}{P^{\prime} \cdot l^{\prime}}=\frac{W^{2}+Q^{2}-m_{N}^{2}}{s-m_{N}^{2}}
\end{aligned}
$$

The identification of the momenta is clear from Eq. (2.1); $m_{N}$ is the nucleon and $m_{\pi}$ the pion mass, respectively. In what follows we neglect the electron mass and the difference of proton and neutron masses.

The differential cross section is equal to

$$
\frac{d \sigma}{d y d Q^{2} d \phi}=\frac{1}{(2 \pi)^{5}} \frac{|\mathcal{M}|^{2}}{64\left(s-m_{N}^{2}\right)} \beta(W) d \phi_{\pi} d(\cos \theta) .
$$

Here $\mathcal{M}$ is the amplitude:

$$
\mathcal{M}=\frac{4 \pi \alpha_{e m}}{q^{2}} \bar{u}(l) \gamma_{\mu} u\left(l^{\prime}\right)\left\langle N \pi\left|j_{\mu}^{e m}(0)\right| p\right\rangle,
$$

$\theta$ and $\phi_{\pi}$ are the polar and azimuthal angles of the pion in the final nucleon-pion c.m. frame, respectively, $\phi$ is the azimuth of the scattered electron in the same frame, the electromagnetic current is defined as

$$
j_{\mu}^{\mathrm{em}}(x)=e_{u} \bar{u}(x) \gamma_{\mu} u(x)+e_{d} \bar{d}(x) \gamma_{\mu} d(x),
$$

and $\beta(W)$ is the kinematic factor related to the c.m.s. momentum of the subprocess $\gamma^{*}(q)+p\left(P^{\prime}\right) \rightarrow$ $\pi(k)+N(P)$ in the final state:

$\vec{k}_{f}^{2}=\frac{W^{2}}{4}\left(1-\frac{\left(m_{N}+m_{\pi}\right)^{2}}{W^{2}}\right)\left(1-\frac{\left(m_{N}-m_{\pi}\right)^{2}}{W^{2}}\right)$,

$\beta(W)=\frac{2\left|\vec{k}_{f}\right|}{W}$.

Alternatively, one could use the Mandelstam $t$-variable (of the $\gamma^{*} p \rightarrow \pi N$ subprocess) $t=\left(P^{\prime}-P\right)^{2}$ :

$$
d t=2\left|\vec{k}_{i}\right|\left|\vec{k}_{f}\right| d(\cos \theta),
$$

where $\vec{k}_{i}$ is the c.m.s. momentum in the initial state:

$$
\vec{k}_{i}^{2}=\frac{W^{2}}{4}\left(1-2 \frac{m_{N}^{2}-Q^{2}}{W^{2}}+\frac{\left(m_{N}^{2}+Q^{2}\right)^{2}}{W^{4}}\right) .
$$

We will be interested in the pion production exactly at threshold, in which case the pion momentum is simply proportional to that of the final state nucleon:

$$
k_{\mu}=\delta P_{\mu}, \quad \delta=m_{\pi} / m_{N} \simeq 0.15,
$$

and assume that the photon virtuality is large, $Q^{2} \gg \Lambda_{\mathrm{Q} C D}^{2}$. The conditions of Lorentz covariance and electromagnetic current conservation imply that the amplitudes at threshold can be parametrized in terms of two generalized form factors which we define as

$$
\left\langle N(P) \pi(k)\left|j_{\mu}^{e m}(0)\right| p\left(P^{\prime}\right)\right\rangle=-\frac{i}{f_{\pi}} \bar{N}(P) \gamma_{5}\left\{\left(\gamma_{\mu} q^{2}-q_{\mu} q\right) \frac{1}{m_{N}^{2}} G_{1}^{\pi N}\left(Q^{2}\right)-\frac{i \sigma_{\mu \nu} q^{\nu}}{2 m_{N}} G_{2}^{\pi N}\left(Q^{2}\right)\right\} N\left(P^{\prime}\right)
$$

Hereafter $N(P)$ is the usual Dirac $u$-spinor, $f_{\pi}=$ $93 \mathrm{MeV}$. For example, the $S$-wave contribution to the structure functions of the total deep-inelastic
(DIS) cross section from unpolarized protons close to the pion-nucleon threshold is given in terms of the generalized form factors as: 


$$
\begin{aligned}
& F_{1}=\frac{\beta(W)}{\left(4 \pi f_{\pi}\right)^{2}} \frac{Q^{2}+\left(2 m_{N}+m_{\pi}\right)^{2}}{2 m_{N}^{3}\left(m_{N}+m_{\pi}\right)}\left(G_{1}^{\pi N} Q^{2}-\frac{1}{2} G_{2}^{\pi N} m_{N} m_{\pi}\right)^{2}, \\
& F_{2}=\frac{\beta(W)}{\left(4 \pi f_{\pi}\right)^{2}} \frac{Q^{2}\left(Q^{2}+m_{\pi}\left(2 m_{N}+m_{\pi}\right)\right)}{m_{N}^{3}\left(m_{N}+m_{\pi}\right)}\left(\left(G_{1}^{\pi N}\right)^{2} Q^{2}+\frac{1}{4}\left(G_{2}^{\pi N}\right)^{2} m_{N}^{2}\right) .
\end{aligned}
$$

The calculation of $G_{1}^{\pi N}\left(Q^{2}\right)$ and $G_{2}^{\pi N}\left(Q^{2}\right)$ in the light-cone sum rule approach presents our main goal.

Having in mind the practical construction of lightcone sum rules that involve nucleon DAs, we define a light-like vector $z_{\mu}$ by the condition

$$
q \cdot z=0, \quad z^{2}=0
$$

and introduce the second light-like vector

$$
p_{\mu}=P_{\mu}-\frac{1}{2} z_{\mu} \frac{m_{N}^{2}}{P \cdot z}, \quad p^{2}=0,
$$

so that $P \rightarrow p$ if the nucleon mass can be neglected, $m_{N} \rightarrow 0$. The photon momentum can be written as

$$
q_{\mu}=q_{\perp \mu}+z_{\mu} \frac{P \cdot q}{P \cdot z} .
$$

We also need the projector onto the directions orthogonal to $p$ and $z$,

$$
g_{\mu \nu}^{\perp}=g_{\mu \nu}-\frac{1}{p z}\left(p_{\mu} z_{\nu}+p_{\nu} z_{\mu}\right),
$$

and use the notation

$$
a_{z} \equiv a_{\mu} z^{\mu}, \quad a_{p} \equiv a_{\mu} p^{\mu},
$$

for arbitrary Lorentz vectors $a_{\mu}$. In turn, $a_{\perp}$ denotes the generic component of $a_{\mu}$ orthogonal to $z$ and $p$, in particular

$$
q_{\perp \mu}=q_{\mu}-\frac{p \cdot q}{p \cdot z} z_{\mu} .
$$

We use the standard Bjorken-Drell convention [39] for the metric and the Dirac matrices; in particular, $\gamma_{5}=i \gamma^{0} \gamma^{1} \gamma^{2} \gamma^{3}$, and the Levi-Civita tensor $\epsilon_{\mu \nu \lambda \sigma}$ is defined as the totally antisymmetric tensor with $\epsilon_{0123}=1$.

Assume for a moment that the nucleon moves in the positive $\mathbf{e}_{\mathbf{z}}$ direction, then $p^{+}$and $z^{-}$are the only nonvanishing components of $p$ and $z$, respectively. The infinite momentum frame can be visualized as the limit $p^{+} \sim Q \rightarrow \infty$ with fixed $P \cdot z=p \cdot z \sim 1$ where $Q$ is the large scale in the process. Expanding the matrix element in powers of $1 / p^{+}$introduces the power counting in $Q$. In this language, twist counts the suppression in powers of $p^{+}$. Similarly, the nucleon spinor $N(P, \lambda)$ has to be decomposed in "large" and "small" components as

$$
\begin{aligned}
N(P, \lambda) & =\frac{1}{2 p \cdot z}(\not \not \not z+\not \not p) N(P, \lambda) \\
& =N^{+}(P, \lambda)+N^{-}(P, \lambda),
\end{aligned}
$$

where we have introduced two projection operators

$$
\Lambda^{+}=\frac{\not p \not z}{2 p \cdot z} \quad, \quad \Lambda^{-}=\frac{\not \not p}{2 p \cdot z}
$$

that project onto the "plus" and "minus" components of the spinor. Note the useful relations

$$
\not p N(P)=m_{N} N^{+}(P), \quad \not k N(P)=\frac{2 p \cdot z}{m_{N}} N^{-}(P)
$$

that are a consequence of the Dirac equation $\not P N(P)=m_{N} N(P)$. Using the explicit expressions for $N(P)$ it is easy to see that $\Lambda^{+} N=N^{+} \sim \sqrt{p^{+}}$ while $\Lambda^{-} N=N^{-} \sim 1 / \sqrt{p^{+}}$.

Note that all expressions are invariant under the reparametrization $z_{\mu} \rightarrow \alpha z_{\mu}$ where $\alpha$ is a real number; we will use this freedom to set $z_{\mu}$ equal to the "minus" component of the distance between the currents in the operator product.

\section{PION NUCLEON GENERALIZED DISTRIBUTION AMPLITUDES}

\section{A. Leading Twist}

Our purpose will be to get a complete classification of three-quark generalized distribution amplitudes (GDAs) of a $n \pi^{+}$and $p \pi^{0}$ pair in the limit that the momentum of the pion relative to that of the nucleon is small which will be a generalization of [40]. This can be done using soft-pion theorems, in the spirit of the work [5]. In order to develop the necessary formalism we first consider the leading twist GDA in some detail. 


\section{Nucleon Distribution Amplitudes}

We begin with quoting the necessary portion of the definitions and results from [40].

The notion of hadron distribution amplitudes
(DAs) in general refers to hadron-to-vacuum matrix elements of nonlocal operators build of quark and gluon fields at light-like separations. In this paper we will deal with the three-quark matrix element

$$
\left\langle 0\left|\epsilon^{i j k} u_{\alpha}^{i^{\prime}}\left(a_{1} z\right)\left[a_{1} z, a_{0} z\right]_{i^{\prime}, i} u_{\beta}^{j^{\prime}}\left(a_{2} z\right)\left[a_{2} z, a_{0} z\right]_{j^{\prime}, j} d_{\gamma}^{k^{\prime}}\left(a_{3} z\right)\left[a_{3} z, a_{0} z\right]_{k^{\prime}, k}\right| p(P, \lambda)\right\rangle
$$

where $|p(P, \lambda)\rangle$ denotes the proton state with momentum $P, P^{2}=M^{2}$ and helicity $\lambda$. $u, d$ are the quark-field operators. The Greek letters $\alpha, \beta, \gamma$ stand for Dirac indices, the Latin letters $i, j, k$ refer to color. $z$ is an arbitrary light-like vector, $z^{2}=0$, the $a_{i}$ are real numbers. The gauge-factors $[x, y]$ are defined as

$$
[x, y]=\mathrm{P} \exp \left[i g \int_{0}^{1} \mathrm{~d} t(x-y)_{\mu} A^{\mu}(t x+(1-t) y)\right]
$$

and render the matrix element in 3.1 gaugeinvariant. To simplify the notation we will not write the gauge-factors explicitly in what follows but imply that they are always present.

Taking into account Lorentz covariance, spin and parity of the nucleon, the most general decomposition of the matrix element in Eq. (3.1) involves 24 invariant functions [40]. To the leading twist-three accuracy only three of them are relevant. We can write in a shorthand notation

$$
\begin{aligned}
& 4\left\langle 0\left|\varepsilon^{i j k} u_{\alpha}^{i}\left(a_{1} z\right) u_{\beta}^{j}\left(a_{2} z\right) d_{\gamma}^{k}\left(a_{3} z\right)\right| p(P, \lambda)\right\rangle_{\mathrm{twist}-3}= \\
& =V_{1}^{p}\left(v_{1}\right)_{\alpha \beta, \gamma}+A_{1}^{p}\left(a_{1}\right)_{\alpha \beta, \gamma}+T_{1}^{p}\left(t_{1}\right)_{\alpha \beta, \gamma}
\end{aligned}
$$

where

$$
\begin{aligned}
& \left(v_{1}\right)_{\alpha \beta, \gamma}=(\not p C)_{\alpha \beta}\left(\gamma_{5} N^{+}\right)_{\gamma} \\
& \left(a_{1}\right)_{\alpha \beta, \gamma}=\left(\not p \gamma_{5} C\right)_{\alpha \beta} N_{\gamma}^{+} \\
& \left(t_{1}\right)_{\alpha \beta, \gamma}=\left(i \sigma_{\perp p} C\right)_{\alpha \beta}\left(\gamma^{\perp} \gamma_{5} N^{+}\right)_{\gamma}
\end{aligned}
$$

stand for the Lorentz structures.

In turn, $V_{1}^{p}, A_{1}^{p}, T_{1}^{p}$ can be written as

$$
F\left(a_{i} p \cdot z\right)=\int \mathcal{D} x e^{-i p z \sum_{i} x_{i} a_{i}} F\left(x_{i}\right),
$$

where the functions $F\left(x_{i}\right)$ depend on the dimensionless variables $x_{i}, 0<x_{i}<1, \sum_{i} x_{i}=1$ which correspond to the longitudinal momentum fractions carried by the quarks inside the nucleon. The integration measure is defined as

$$
\int \mathcal{D} x=\int_{0}^{1} \mathrm{~d} x_{1} \mathrm{~d} x_{2} \mathrm{~d} x_{3} \delta\left(x_{1}+x_{2}+x_{3}-1\right) .
$$

Note that

$$
\begin{aligned}
& \left(v_{1}\right)_{\alpha \beta, \gamma}=\left(v_{1}\right)_{\beta \alpha, \gamma}, \\
& \left(a_{1}\right)_{\alpha \beta, \gamma}=-\left(a_{1}\right)_{\beta \alpha, \gamma}, \\
& \left(t_{1}\right)_{\alpha \beta, \gamma}=\left(t_{1}\right)_{\beta \alpha, \gamma} .
\end{aligned}
$$

Since the operator on the l.h.s. of Eq. (3.3) is symmetric under the exchange of the two $u$-quarks, this property implies that the $V$ - and $T$-functions are symmetric and the $A$-function is antisymmetric under the exchange of the first two arguments, respectively:

$$
\begin{aligned}
& V^{p}(1,2,3)=V^{p}(2,1,3), \\
& A^{p}(1,2,3)=-A^{p}(2,1,3), \\
& T^{p}(1,2,3)=T^{p}(2,1,3) .
\end{aligned}
$$

In addition, the matrix element in Eq. (3.3) has to fulfill the symmetry relation

$$
\begin{aligned}
& \left\langle 0\left|\varepsilon^{i j k} u_{\alpha}^{i}(1) u_{\beta}^{j}(2) d_{\gamma}^{k}(3)\right| P\right\rangle \\
& +\left\langle 0\left|\varepsilon^{i j k} u_{\alpha}^{i}(1) u_{\gamma}^{j}(3) d_{\beta}^{k}(2)\right| P\right\rangle \\
& +\left\langle 0\left|\varepsilon^{i j k} u_{\gamma}^{i}(3) u_{\beta}^{j}(2) d_{\alpha}^{k}(1)\right| P\right\rangle=0
\end{aligned}
$$

that follows from the condition that the nucleon state has isospin 1/2:

$$
\left(T^{2}-\frac{1}{2}\left(\frac{1}{2}+1\right)\right)\left\langle 0\left|\varepsilon^{i j k} u_{\alpha}^{i}(1) u_{\beta}^{j}(2) d_{\gamma}^{k}(3)\right| P\right\rangle=0,
$$

where

$$
T^{2}=\frac{1}{2}\left(T_{+} T_{-}+T_{-} T_{+}\right)+T_{3}^{2}
$$


and $T_{ \pm}$are the usual isospin step-up and step-down operators. Applying the set of Fierz transformations

$$
\begin{aligned}
& \left(v_{1}\right)_{\gamma \beta, \alpha}=\frac{1}{2}\left(v_{1}-a_{1}-t_{1}\right)_{\alpha \beta, \gamma} \\
& \left(a_{1}\right)_{\gamma \beta, \alpha}=\frac{1}{2}\left(-v_{1}+a_{1}-t_{1}\right)_{\alpha \beta, \gamma} \\
& \left(t_{1}\right)_{\gamma \beta, \alpha}=-\left(v_{1}+a_{1}\right)_{\alpha \beta, \gamma}
\end{aligned}
$$

one ends up with the condition

$2 T_{1}^{p}(1,2,3)=\left[V_{1}^{p}-A_{1}^{p}\right](1,3,2)+\left[V_{1}^{p}-A_{1}^{p}\right](2,3,1)$,

which allows to express the tensor DA of the leading twist in terms of the vector and axial vector distributions. Since the latter have different symmetry, they can be combined together to define the single independent leading twist-3 proton DA

$$
\Phi_{3}^{p}\left(x_{1}, x_{2}, x_{3}\right)=\left[V_{1}^{p}-A_{1}^{p}\right]\left(x_{1}, x_{2}, x_{3}\right)
$$

which is well known and received a lot of attention in the literature. The neutron leading twist DA $\Phi_{3}^{n}\left(x_{1}, x_{2}, x_{3}\right)$ can readily be obtained by the interchange of $u$ and $d$ quarks in the defining Eq. (3.3). For all invariant functions $F=V, A, T$ proton and neutron DAs differ by an overall sign:

$$
F^{p}(1,2,3)=-F^{n}(1,2,3)
$$

as follows from the isospin symmetry. This property is retained for all twists.

\section{Pion-Nucleon Generalized Distribution Amplitudes of twist-3}

Our aim is to describe the generalized distribution amplitudes (GDA) of a pion-nucleon system with small invariant mass in the similar formalism. To this end we define for the $p \pi^{0}$-system:

$$
4\left\langle 0\left|\varepsilon^{i j k} u_{\alpha}^{i}\left(a_{1} z\right) u_{\beta}^{j}\left(a_{2} z\right) d_{\gamma}^{k}\left(a_{3} z\right)\right| p(P, \lambda) \pi^{0}\right\rangle_{\mathrm{tw}-3}=\left(\gamma_{5}\right)_{\gamma \delta} \frac{-i}{f_{\pi}}\left[V_{1}^{p \pi^{0}}\left(v_{1}\right)_{\alpha \beta, \delta}+A_{1}^{p \pi^{0}}\left(a_{1}\right)_{\alpha \beta, \delta}+T_{1}^{p \pi^{0}}\left(t_{1}\right)_{\alpha \beta, \delta}\right]
$$

and, similar, for $n \pi^{+}$:

$4\left\langle 0\left|\varepsilon^{i j k} u_{\alpha}^{i}\left(a_{1} z\right) u_{\beta}^{j}\left(a_{2} z\right) d_{\gamma}^{k}\left(a_{3} z\right)\right| n(P, \lambda) \pi^{+}\right\rangle_{\mathrm{tw}-3}=\left(\gamma_{5}\right)_{\gamma \delta} \frac{-i}{f_{\pi}}\left[V_{1}^{n \pi^{+}}\left(v_{1}\right)_{\alpha \beta, \delta}+A_{1}^{n \pi^{+}}\left(a_{1}\right)_{\alpha \beta, \delta}+T_{1}^{n \pi^{+}}\left(t_{1}\right)_{\alpha \beta, \delta}\right]$.

An extra $\gamma_{5}$ is needed to conserve parity. Similar to the proton case, the symmetry of the two $u$-quarks implies that the GDAs $V$ and $T$ are symmetric, and $A$ is antisymmetric to the exchange of the first two arguments, respectively.

As observed in [5], the GDAs can be calculated using current algebra for small invariant masses of the pion-nucleon system in the soft pion limit. One obtains

$$
\left\langle 0|O| \pi^{a}(k) N_{f}(P, \lambda)\right\rangle=-\frac{i}{f_{\pi}}\left\langle 0\left|\left[Q_{5}^{a}, O\right]\right| N_{f}(P, \lambda)\right\rangle-\frac{i g_{A}}{4 f_{\pi}(P \cdot k)} \sum_{\lambda^{\prime}, f^{\prime}}\left\langle 0|O| N_{f^{\prime}}\left(P, \lambda^{\prime}\right)\right\rangle \bar{N}\left(P, \lambda^{\prime}\right) \not k \gamma_{5} \tau_{f^{\prime} f}^{a} N(P, \lambda)
$$

and similar for the pion-nucleon final state

$$
\left\langle\pi^{a}(k) N_{f}(P, \lambda)\left|O^{\dagger}\right| 0\right\rangle=-\frac{i}{f_{\pi}}\left\langle N_{f}(P, \lambda)\left|\left[Q_{5}^{a}, O^{\dagger}\right]\right| 0\right\rangle+\frac{i g_{A}}{4 f_{\pi}(P \cdot k)} \sum_{\lambda^{\prime}, f^{\prime}} \bar{N}(P, \lambda) \not k \gamma_{5} \tau_{f f^{\prime}}^{a} N\left(P, \lambda^{\prime}\right)\left\langle N_{f^{\prime}}\left(P, \lambda^{\prime}\right)\left|O^{\dagger}\right| 0\right\rangle .
$$

Here $f_{\pi}=93 \mathrm{MeV}$ is the pion decay constant defined as $\left\langle 0\left|\bar{q} \gamma_{\mu} \gamma_{5} \frac{1}{2} \tau^{a} q\right| \pi^{b}(k)\right\rangle=i \delta^{a b} f_{\pi} k_{\mu}$ and $g_{A} \simeq 1.25$ is the axial charge of the nucleon. $O$ is the nonlocal three-quark operator and $Q_{5}^{a}$ is the operator of the axial charge

$$
Q_{5}^{a}=\int d^{3} x \bar{q}(x) \gamma_{0} \gamma_{5} \frac{\tau^{a}}{2} q(x), \quad q=\left(\begin{array}{l}
\mathrm{u} \\
\mathrm{d}
\end{array}\right)
$$


where the $\tau^{a}$ are the usual Pauli matrices. The second term in Eq. (3.18), Eq. (3.19) corresponds to the pion bremsstrahlung from the ingoing (outgoing) nucleon. This term corresponds to a $\pi N$ system in $\mathrm{P}$-wave and can, in principle, be separated from the first (commutator) contribution that is Swave by considering the angular distributions in the $\pi N$ system. Note also that the bremsstrahlung contribution vanishes at the threshold of pion production $W_{\mathrm{th}}=M+m_{\pi}$ but becomes significant for $W-W_{\mathrm{th}} \sim m_{\pi}$. This contribution is determined in terms of the nucleon DAs that eventually combine to produce the nucleon electromagnetic form factors; it can always be added. In what follows we will concentrate on the S-wave (commutator) term.

For our purposes we need to specify $O$ to be the relevant three-local light-ray operator.

$$
O \rightarrow O_{\alpha \beta \gamma}^{u u d}=\varepsilon^{i j k} u_{\alpha}^{i}\left(a_{1} z\right) u_{\beta}^{j}\left(a_{2} z\right) d_{\gamma}^{k}\left(a_{3} z\right)
$$

Here the superscript (uud) indicates the flavor content and simultaneously the order in which the quark fields are positioned on the light ray. For example, $O_{\alpha \beta \gamma}^{u u d}$ and $O_{\alpha \beta \gamma}^{u d u}$ correspond to the $u_{\alpha}\left(a_{1} z\right) u_{\beta}\left(a_{2} z\right) d_{\gamma}\left(a_{3} z\right)$ and $u_{\alpha}\left(a_{1} z\right) d_{\beta}\left(a_{2} z\right) u_{\gamma}\left(a_{3} z\right)$ configurations, respectively. One obtains

$$
\begin{aligned}
\left\langle 0\left|O_{\alpha \beta \gamma}^{u u d}\right| n(P, \lambda) \pi^{+}(k)\right\rangle & =\frac{1}{\sqrt{2}}\left\{\left\langle 0\left|O_{\alpha \beta \gamma}^{u u d}\right| n(P, \lambda) \pi^{1}(k)\right\rangle+i\left\langle 0\left|O_{\alpha \beta \gamma}^{u u d}\right| n(P, \lambda) \pi^{2}(k)\right\rangle\right\} \\
& =-\frac{i}{\sqrt{2} f_{\pi}}\left\{\left\langle 0\left|\left[Q_{5}^{1}, O_{\alpha \beta \gamma}^{u u d}\right]\right| n(P, \lambda)\right\rangle+i\left\langle 0\left|\left[Q_{5}^{2}, O_{\alpha \beta \gamma}^{u u d}\right]\right| n(P, \lambda)\right\rangle\right\}+\ldots \\
\left\langle 0\left|O_{\alpha \beta \gamma}^{u u d}\right| p(P, \lambda) \pi^{0}(k)\right\rangle & =-\frac{i}{f_{\pi}}\left\langle 0\left|\left[Q_{5}^{3}, O_{\alpha \beta \gamma}^{u u d}\right]\right| p(P, \lambda)\right\rangle+\ldots
\end{aligned}
$$

where the ellipses stand for bremsstrahlung contributions. Calculation of the commutators $\left[Q_{5}^{1}, O_{\alpha \beta \gamma}^{u u d}(y)\right]$ and $\left[Q_{5}^{3}, O_{\alpha \beta \gamma}^{u u d}(y)\right]$ reduces to a chiral rotation

$$
\left[Q_{5}^{a}, q_{f}\right]=-\left(\frac{\tau^{a}}{2}\right)_{f f^{\prime}} \gamma_{5} q_{f^{\prime}} .
$$

For the three-quark operators one uses the chain rule $[A, B C D]=[A, B] C D+B[A, C] D+B C[A, D]$ to obtain:

$$
\begin{aligned}
{\left[Q_{5}^{1}, O_{\alpha \beta \gamma}^{u u d}\right] } & =-\frac{1}{2}\left\{\left(\gamma_{5}\right)_{\alpha \lambda} O_{\lambda \gamma \beta}^{d d u}+\left(\gamma_{5}\right)_{\beta \lambda} O_{\lambda \gamma \alpha}^{d d u}+\left(\gamma_{5}\right)_{\gamma \lambda} O_{\alpha \beta \lambda}^{u u u}\right\}, \\
{\left[Q_{5}^{2}, O_{\alpha \beta \gamma}^{u u d}\right] } & =\frac{i}{2}\left\{\left(\gamma_{5}\right)_{\alpha \lambda} O_{\lambda \gamma \beta}^{d d u}+\left(\gamma_{5}\right)_{\beta \lambda} O_{\lambda \gamma \alpha}^{d d u}-\left(\gamma_{5}\right)_{\gamma \lambda} O_{\alpha \beta \lambda}^{u u u}\right\}, \\
{\left[Q_{5}^{3}, O_{\alpha \beta \gamma}^{u u d}\right] } & =-\frac{1}{2}\left\{\left(\gamma_{5}\right)_{\alpha \lambda} O_{\lambda \beta \gamma}^{u u d}+\left(\gamma_{5}\right)_{\beta \lambda} O_{\alpha \lambda \gamma}^{u u d}-\left(\gamma_{5}\right)_{\gamma \lambda} O_{\alpha \beta \lambda}^{u u d}\right\} .
\end{aligned}
$$

Taking the nucleon-to-vacuum matrix element, Eqs. (3.3), (3.16) and (3.17), and using symmetry relations (3.7) and Fierz transformations (3.12) one obtains after some algebra

$$
\begin{aligned}
& A_{1}^{n \pi^{+}}(1,2,3)=\frac{1}{\sqrt{2}}\left\{\frac{1}{2} V_{1}^{n}(1,3,2)-\frac{1}{2} V_{1}^{n}(2,3,1)-\frac{1}{2} A_{1}^{n}(2,3,1)+\frac{1}{2} A_{1}^{n}(1,3,2)+T_{1}^{n}(2,3,1)-T_{1}^{n}(1,3,2)\right\}, \\
& V_{1}^{n \pi^{+}}(1,2,3)=\frac{1}{\sqrt{2}}\left\{\frac{1}{2} V_{1}^{n}(1,3,2)+\frac{1}{2} A_{1}^{n}(1,3,2)+T_{1}^{n}(1,3,2)+\frac{1}{2} V_{1}^{n}(2,3,1)+\frac{1}{2} A_{1}^{n}(2,3,1)+T_{1}^{n}(2,3,1)\right\} \text {, } \\
& T_{1}^{n \pi^{+}}(1,2,3)=\frac{1}{2 \sqrt{2}}\left\{A_{1}^{n}(2,3,1)+A_{1}^{n}(1,3,2)-V_{1}^{n}(2,3,1)-V_{1}^{n}(1,3,2)\right\},
\end{aligned}
$$

and

$$
V_{1}^{p \pi^{0}}(1,2,3)=\frac{1}{2} V_{1}^{p}(1,2,3),
$$




$$
\begin{aligned}
& A_{1}^{p \pi^{0}}(1,2,3)=\frac{1}{2} A_{1}^{p}(1,2,3), \\
& T_{1}^{p \pi^{0}}(1,2,3)=\frac{3}{2} T_{1}^{p}(1,2,3),
\end{aligned}
$$

which is the desired result. One can further simplify (3.25) using the substitution in Eq. (3.13) to obtain:

$$
\begin{aligned}
& V_{1}^{n \pi^{+}}(1,2,3)=\frac{1}{\sqrt{2}}\left\{V_{1}^{n}(1,3,2)+V_{1}^{n}(1,2,3)+V_{1}^{n}(2,3,1)+A_{1}^{n}(1,3,2)+A_{1}^{n}(2,3,1)\right\}, \\
& A_{1}^{n \pi^{+}}(1,2,3)=-\frac{1}{\sqrt{2}}\left\{V_{1}^{n}(3,2,1)-V_{1}^{n}(1,3,2)+A_{1}^{n}(2,1,3)+A_{1}^{n}(2,3,1)+A_{1}^{n}(3,1,2)\right\}, \\
& T_{1}^{n \pi^{+}}(1,2,3)=\frac{1}{2 \sqrt{2}}\left\{A_{1}^{n}(2,3,1)+A_{1}^{n}(1,3,2)-V_{1}^{n}(2,3,1)-V_{1}^{n}(1,3,2)\right\} .
\end{aligned}
$$

Note that the functions $V, A, T$ for the both cases $n \pi^{+}$and $p \pi^{0}$ satisfy the symmetry conditions (3.8) which follow directly from their definitions in (3.16), (3.16), respectively. On the other hand, the isospin relation similar to (3.13) is not valid, since the pionnucleon pair can have both isospin $1 / 2$ and $3 / 2$.

\section{B. Higher Twists}

Taking into account Lorentz covariance, spin and parity of the nucleon and the pion, the most general decomposition of the three-quark matrix element in Eq. (3.1) involves 24 invariant functions. In total, there are three leading-twist3 invariant functions $\left(V_{1}, A_{1}, T_{1}\right)$, nine of twist$4\left(V_{2}, A_{2}, V_{3}, A_{3}, S_{1}, P_{1}, T_{2}, T_{3}, T_{7}\right)$, nine of twist-5 $\left(V_{4}, A_{4}, V_{5}, A_{5}, S_{2}, P_{2}, T_{4}, T_{5}, T_{8}\right)$, and three of twist$6\left(V_{6}, A_{6}, T_{6}\right)$. Using the shorthand notation of Ref. [40] for the relevant Lorentz structures, we define

$$
\begin{aligned}
& 4 \cdot\left\langle 0\left|\varepsilon^{i j k} u_{\alpha}^{i}\left(a_{1} z\right) u_{\beta}^{j}\left(a_{2} z\right) d_{\gamma}^{k}\left(a_{3} z\right)\right| N(P, \lambda) \pi(k)\right\rangle= \\
&=\left(\gamma_{5}\right)_{\gamma \delta} \frac{-i}{f_{\pi}}\left[S_{1}^{\pi N}\left(s_{1}\right)_{\alpha \beta, \delta}+S_{2}^{\pi N}\left(s_{2}\right)_{\alpha \beta, \delta}+P_{1}^{\pi N}\left(p_{1}\right)_{\alpha \beta, \delta}+P_{2}^{\pi N}\left(p_{2}\right)_{\alpha \beta, \delta}\right. \\
&+V_{1}^{\pi N}\left(v_{1}\right)_{\alpha \beta, \delta}+V_{2}^{\pi N}\left(v_{2}\right)_{\alpha \beta, \delta}+\frac{1}{2} V_{3}^{\pi N}\left(v_{3}\right)_{\alpha \beta, \delta}+\frac{1}{2} V_{4}^{\pi N}\left(v_{4}\right)_{\alpha \beta, \delta} \\
&+V_{5}^{\pi N}\left(v_{5}\right)_{\alpha \beta, \delta}+V_{6}^{\pi N}\left(v_{6}\right)_{\alpha \beta, \delta}+A_{1}^{\pi N}\left(a_{1}\right)_{\alpha \beta, \delta}+A_{2}^{\pi N}\left(a_{2}\right)_{\alpha \beta, \delta} \\
&+\frac{1}{2} A_{3}^{\pi N}\left(a_{3}\right)_{\alpha \beta, \delta}+\frac{1}{2} A_{4}^{\pi N}\left(a_{4}\right)_{\alpha \beta, \delta}+A_{5}^{\pi N}\left(a_{5}\right)_{\alpha \beta, \delta}+A_{6}^{\pi N}\left(a_{6}\right)_{\alpha \beta, \delta} \\
&+T_{1}^{\pi N}\left(t_{1}\right)_{\alpha \beta, \delta}+T_{2}^{\pi N}\left(t_{2}\right)_{\alpha \beta, \delta}+T_{3}^{\pi N}\left(t_{3}\right)_{\alpha \beta, \delta}+T_{4}^{\pi N}\left(t_{4}\right)_{\alpha \beta, \delta} \\
&+\left.T_{5}^{\pi N}\left(t_{5}\right)_{\alpha \beta, \delta}+T_{6}^{\pi N}\left(t_{6}\right)_{\alpha \beta, \delta}+\frac{1}{2} T_{7}^{\pi N}\left(t_{7}\right)_{\alpha \beta, \delta}+\frac{1}{2} T_{8}^{\pi N}\left(t_{8}\right)_{\alpha \beta, \delta}\right] .
\end{aligned}
$$

The calculation of higher-twist pion-nucleon GDAs is similar to the leading twist, but proves to be much more cumbersome. The complete set of the necessary Fierz identities for the Lorentz structures 
$\left(v_{i}\right)_{\alpha \beta, \delta},\left(a_{i}\right)_{\alpha \beta, \delta}\left(t_{i}\right)_{\alpha \beta, \delta}$ is given in Ref. [40]. The results for the GDAs of all twists are collected in Appendix A below. In addition, in Appendix B we present the results for the functions $\mathcal{V}_{1}^{M}$ and $\mathcal{A}_{1}^{M}$ that appear in the off-light-cone $\mathcal{O}\left(x^{2}\right)$ corrections in the OPE involving three-quark currents,

$$
\begin{aligned}
& 4\left\langle 0\left|\varepsilon^{i j k} u_{\alpha}^{i}\left(a_{1} x\right) u_{\beta}^{j}\left(a_{2} x\right) d_{\gamma}^{k}\left(a_{3} x\right)\right| N \pi\right\rangle= \\
& =\left(\gamma_{5}\right)_{\gamma \delta} \frac{-i}{f_{\pi}}\left\{\left[V_{1}+\frac{x^{2} m_{N}^{2}}{4} \mathcal{V}_{1}^{M}\right]\left(v_{1}\right)_{\alpha \beta, \delta}\right. \\
& +\left[A_{1}+\frac{x^{2} m_{N}^{2}}{4} \mathcal{A}_{1}^{M}\right]\left(a_{1}\right)_{\alpha \beta, \delta} \\
& \left.+\left[T_{1}+\frac{x^{2} m_{N}^{2}}{4} \mathcal{T}_{1}^{M}\right]\left(t_{1}\right)_{\alpha \beta, \delta}\right\}^{\pi N}+\ldots,
\end{aligned}
$$

for the case that the light-cone positions of two of the three quarks coincide, to the first subleading order in the conformal expansion. The function $\mathcal{T}_{1}^{M}$ does not contribute to the sum rules to our accuracy.

\section{LIGHT CONE SUM RULES}

\section{A. The soft pion limit}

For technical reasons, it is convenient to write the sum rules for the complex conjugated amplitude with the pion-nucleon pair in the initial state. To this end we consider the correlation function

$$
\begin{aligned}
& T_{\nu}^{\pi N}(P, q)= \\
& =i \int \mathrm{d}^{4} x e^{i q x}\left\langle 0\left|T\left\{\eta_{p}(0) j_{\nu}^{\mathrm{em}}(x)\right\}\right| N(P) \pi(k)\right\rangle,
\end{aligned}
$$

where

$$
\begin{aligned}
& \eta_{p}(x)=\varepsilon^{i j k}\left[u^{i}(x) C \gamma_{\mu} u^{j}(x)\right] \gamma_{5} \gamma^{\mu} d^{k}(x), \\
& \left\langle 0\left|\eta_{p}(0)\right| N(P)\right\rangle=\lambda_{1}^{p} m_{N} N(P),
\end{aligned}
$$

is the so-called Ioffe proton interpolating current [41], $\lambda_{1}^{p}$ is the corresponding coupling.

In the limit $|k| \rightarrow 0$ for fixed $q^{2}$ and $\left(P^{\prime}\right)^{2}=$ $(P+k-q)^{2}$ the correlation function in (4.1) can be calculated using PCAC and current algebra in terms of the correlation functions without a pion and involving chirally-rotated currents

$$
\begin{aligned}
& T_{\nu}^{\pi N}(P, q)= \\
& =-\frac{i}{f_{\pi}}\left[i \int d^{4} x e^{i q x}\left\langle 0\left|T\left\{\left[Q_{5}^{a}, \eta_{p}(0)\right] j_{\nu}^{\mathrm{em}}(x)\right\}\right| N(P)\right\rangle\right. \\
& \left.\quad+i \int d^{4} x e^{i q x}\left\langle 0\left|T\left\{\eta_{p}(0)\left[Q_{5}^{a}, j_{\nu}^{\mathrm{em}}(x)\right]\right\}\right| N(P)\right\rangle\right]
\end{aligned}
$$

+ bremsstrahlung terms,

where $Q_{5}^{a}$ is the axial charge and the bremsstrahlung contributions correspond to pion absorption by the initial state nucleon.

The commutators can easily be evaluated with the result

$$
\begin{aligned}
& {\left[Q_{5}^{+}, j_{\nu}^{\mathrm{em}}(x)\right]=-\frac{1}{\sqrt{2}} A_{\nu}(x), \quad A_{\nu}=\frac{1}{\sqrt{2}} \bar{q} \gamma_{\nu} \gamma_{5} \tau^{+} q,} \\
& {\left[Q_{5}^{3}, j_{\nu}^{\mathrm{em}}(x)\right]=0}
\end{aligned}
$$

where $\tau^{ \pm}=\frac{1}{\sqrt{2}}\left(\tau^{1} \pm i \tau^{2}\right), Q_{5}^{ \pm}=\frac{1}{\sqrt{2}}\left(Q_{5}^{1} \pm i Q_{5}^{2}\right)$ etc. Also

$$
\begin{gathered}
{\left[Q_{5}^{+}, \eta_{p}(x)\right]=-\frac{1}{\sqrt{2}} \gamma_{5} \eta_{n}(x),} \\
{\left[Q_{5}^{3}, \eta_{p}(x)\right]=-\frac{1}{2} \gamma_{5} \eta_{p}(x),}
\end{gathered}
$$

where $\eta_{n}$ is the neutron current

$$
\eta_{n}(x)=-\varepsilon^{i j k}\left[d^{i}(x) C \gamma_{\mu} d^{j}(x)\right] \gamma_{5} \gamma^{\mu} u^{k}(x) .
$$

The contributions of interest to (4.1) are those sin-
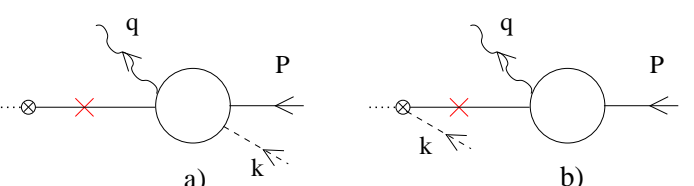

b)

FIG. 2: Schematic structure of the pole terms in the correlation function (4.1)

gular in the vicinity of $P^{\prime 2} \rightarrow m_{N}^{2}$, see Fig. 2, Note that in addition to the nucleon pole, Fig. 2 a, one has to take into account the semidisconnected contribution with the pion-nucleon intermediate state, Fig. 2b. For example, for $n \pi^{+}$one obtains

$$
T_{\nu}^{\pi^{+} n}(P, q)=\frac{i \lambda_{1}^{p} m_{N}}{f_{\pi}} \frac{m_{N}+P^{\prime}}{m_{N}^{2}-P^{\prime 2}} \gamma_{5}\left\{\left(\gamma_{\nu} q^{2}-q_{\nu} \not\right) \frac{G_{1}^{n \pi^{+}}}{m_{N}^{2}}-\frac{i \sigma_{\nu \mu} q^{\mu}}{2 m_{N}} G_{2}^{n \pi^{+}}\right\} N(P)
$$




$$
-\frac{i \lambda_{n \pi}+m_{N}}{f_{\pi}} \gamma_{5} \frac{m_{N}+\not P^{\prime}-\not k}{m_{N}^{2}-\left(P^{\prime}-k\right)^{2}}\left\{\gamma_{\nu} F_{1}^{n}-\frac{i \sigma_{\nu \mu} q^{\mu}}{2 m_{N}} F_{2}^{n}\right\} N(P)+\ldots
$$

where the ellipses stand for less singular contributions, $F_{1}^{n}$ and $F_{2}^{n}$ are Dirac and Pauli electromagnetic neutron form factors, respectively

$$
\left\langle N\left(P^{\prime}\right)\left|j_{\mu}^{\mathrm{em}}(0)\right| N(P)\right\rangle=\bar{N}\left(P^{\prime}\right)\left[\gamma_{\mu} F_{1}^{N}\left(Q^{2}\right)-i \frac{\sigma_{\mu \nu} q^{\nu}}{2 m_{N}} F_{2}^{N}\left(Q^{2}\right)\right] N(P)
$$

and $\lambda_{n \pi^{+}}$is the coupling of the Ioffe current to the $n \pi^{+}$state:

$$
\left\langle 0\left|\eta_{p}(0)\right| N\left(P^{\prime}-k\right) \pi(k)\right\rangle=-\frac{i}{f_{\pi}} \lambda_{\pi N} m_{N} \gamma_{5} N\left(P^{\prime}-k\right) .
$$

On the other hand, using the representation in Eq. (4.3) instead, one obtains

$$
\begin{aligned}
T_{\nu}^{\pi^{+} n}(P, q)= & \frac{i \lambda_{1}^{p} m_{N}}{f_{\pi}} \frac{1}{\sqrt{2}} \frac{m_{N}+P^{\prime}}{m_{N}^{2}-P^{\prime 2}}\left\{\gamma_{\nu} G_{A}\left(Q^{2}\right)-\frac{q_{\nu}}{2 m_{N}} G_{P}\left(Q^{2}\right)-i \frac{\sigma_{\nu \mu} q^{\mu}}{2 m_{N}} G_{T}\left(Q^{2}\right)\right\} \gamma_{5} N(P) \\
& +\frac{i}{\sqrt{2} f_{\pi}} \gamma_{5} \lambda_{1}^{n} m_{N} \frac{m_{N}+P^{\prime}}{m_{N}^{2}-P^{\prime 2}}\left\{\gamma_{\nu} F_{1}^{n}-\frac{i \sigma_{\nu \mu} q^{\mu}}{2 m_{N}} F_{2}^{n}\right\} N(P)+\ldots
\end{aligned}
$$

where the form factors in the first line are defined as

$$
\left\langle N\left(P^{\prime}\right)\left|A_{\nu}(0)\right| N(P)\right\rangle=\bar{N}\left(P^{\prime}\right)\left[\gamma_{\nu} G_{A}\left(Q^{2}\right)-\frac{q_{\nu}}{2 m_{N}} G_{P}\left(Q^{2}\right)-i \frac{\sigma_{\nu \mu} q^{\mu}}{2 m_{N}} G_{T}\left(Q^{2}\right)\right] \gamma_{5} N(P) .
$$

Note that $G_{T}\left(Q^{2}\right)=0$ because of the isospin and $\mathrm{CP}$ invariance.

In the same approximation the pion-nucleon coupling is given by $\lambda_{n \pi^{+}}=\left(-\frac{1}{\sqrt{2}}\right) \lambda_{1}^{p}$ (cf. Eq. 44.5) so that the terms in the second line in Eqs. 4.7) and (4.10) coincide: The contribution of the chiral rotation of the nucleon current is identically equal to the semidisconnected contribution in Fig. 20 of the pion coupling to the current. Equating the remaining contributions in the first line of Eqs. (4.7) and 4.10) one obtains

$$
\begin{aligned}
Q^{2} G_{1}^{n \pi^{+}}\left(Q^{2}\right) & =\frac{m_{N}^{2}}{\sqrt{2}} G_{A}\left(Q^{2}\right)+\mathcal{O}\left(m_{\pi} / \Lambda,|k| / \Lambda\right), \\
G_{2}^{n \pi^{+}}\left(Q^{2}\right) & =0+\mathcal{O}\left(m_{\pi} / \Lambda,|k| / \Lambda\right),
\end{aligned}
$$

which is the classical result [1, 2, 3, 44]. The consideration of the $p \pi^{0}$ state is similar, the only difference being that the commutator of the electromagnetic current with the axial charge vanishes in this case, so that the both amplitudes vanish at threshold, up to corrections in $m_{\pi}$ and/or $|k|$ :

$$
\begin{aligned}
Q^{2} G_{1}^{p \pi^{0}}\left(Q^{2}\right) & =0+\mathcal{O}\left(m_{\pi} / \Lambda,|k| / \Lambda\right), \\
G_{2}^{p \pi^{0}}\left(Q^{2}\right) & =0+\mathcal{O}\left(m_{\pi} / \Lambda,|k| / \Lambda\right) .
\end{aligned}
$$

To avoid misunderstanding, note that our (schematic) derivation does not include bremsstrahlung contributions, which have to be added. The derivation breaks down for large momentum transfers, however. One way to see this is that the limit $k \rightarrow 0$ that is implied in the current algebra relations, becomes far away from the physical region. In particular, the position of the pole in the pion-nucleon intermediate state in Fig. 2 $\mathrm{b}\left(P^{\prime}-k\right)^{2}=m_{N}^{2}$ moves away from the nucleon pole at $\left(P^{\prime}\right)^{2}=m_{N}^{2}$. Requiring that $\left(P^{\prime}-k\right)^{2}-\left(P^{\prime}\right)^{2} \ll \Lambda_{\mathrm{Q} C D}^{2} \sim m_{N}^{2}$ one obtains a restriction for the applicability of the soft pion limit $Q^{2} \ll \Lambda^{3} / m_{\pi}$. In what follows we develop a technique to calculate the amplitudes in the opposite limit of large momentum transfers, $Q^{2} \gg \Lambda^{3} / m_{\pi}$.

\section{B. The sum rules}

The general philosophy of the LCSR approach is explained in the Introduction. The main idea is to separate contributions of small distances to the correlation function in (4.1) in terms of calculable coefficient functions in front of operator matrix elements and apply the soft pion techniques for the evaluation of the latter rather than the correlation function itself. Information about the generalized form factors can then be extracted by matching the QCD calcu- 
lation at moderate negative values $P^{\prime 2} \sim-1 \mathrm{GeV}^{2}$ with the dispersion representation (4.7) in terms of hadronic states.

The correlation function in (4.1) involves several invariant functions that can be separated by the appropriate Lorentz projections. The structures that are most useful for writing the LCSRs are usually those containing the maximum power of the large momentum $p^{+} \sim p z$. We define

$$
\Lambda_{+} T_{z}=+\frac{i}{f_{\pi}}(p z+k z) \gamma_{5}\left\{m_{N} \mathcal{A}+\not q_{\perp} \mathcal{B}\right\} N^{+}(P) .
$$

The invariant functions $\mathcal{A}$ and $\mathcal{B}$ can be calculated for sufficiently large Euclidean $Q^{2}$ and $P^{\prime 2}=$ $(P+k-q)^{2}$ in terms of the generalized distribution amplitudes of the pion-nucleon system using the operator product expansion. We use the following notations:

$$
\begin{aligned}
& \widetilde{F}\left(x_{3}\right)=\int_{1}^{x_{3}} d x_{3}^{\prime} \int_{0}^{1-x_{3}^{\prime}} d x_{1} F\left(x_{1}, 1-x_{1}-x_{3}^{\prime}, x_{3}^{\prime}\right), \\
& \widetilde{F}\left(x_{3}\right)=\int_{1}^{x_{3}} d x_{3}^{\prime} \int_{1}^{x_{3}^{\prime}} d x_{3}^{\prime \prime}
\end{aligned}
$$

$$
\times \int_{0}^{1-x_{3}^{\prime \prime}} d x_{1} F\left(x_{1}, 1-x_{1}-x_{3}^{\prime \prime}, x_{3}^{\prime \prime}\right)
$$

and

$$
\begin{aligned}
\widehat{F}\left(x_{2}\right) & =\int_{1}^{x_{2}} d x_{2}^{\prime} \int_{0}^{1-x_{2}^{\prime}} d x_{1} F\left(x_{1}, x_{2}^{\prime}, 1-x_{1}-x_{2}^{\prime}\right), \\
\widehat{F}\left(x_{2}\right) & =\int_{1}^{x_{2}} d x_{2}^{\prime} \int_{1}^{x_{2}^{\prime}} d x_{2}^{\prime \prime} \\
& \times \int_{0}^{1-x_{2}^{\prime \prime}} d x_{1} F\left(x_{1}, x_{2}^{\prime \prime}, 1-x_{1}-x^{\prime \prime}\right),
\end{aligned}
$$

where $F=A, V, T$ is a generic pion-nucleon GDA that depends on the three valence quark momentum fractions.

To the three-level accuracy $\mathcal{A}^{\pi N}$ and $\mathcal{B}^{\pi N}$ are given by the same expressions [34] as the LCSRs for the proton electromagnetic form factors $F_{1}$ and $F_{2}$, respectively, with the substitution of the nucleon DAs by the pion-nucleon ones:

$$
\begin{aligned}
\mathcal{A}^{\pi N}= & 2 e_{d} \int_{0}^{1} d x_{3}\left\{\frac{Q^{2}+q_{3}^{2}}{q_{3}^{4}} \widetilde{V}_{123}^{\pi N}+\frac{x_{3}}{q_{3}^{2}} \int_{0}^{\bar{x}_{3}} d x_{1} V_{3}^{\pi N}\left(x_{i}\right)+\frac{x_{3}^{2} m_{N}^{2}}{q_{3}^{4}} \widetilde{V}_{43}^{\pi N}\right\} \\
& +2 e_{u} \int_{0}^{1} d x_{2}\left\{\frac{x_{2}}{q_{2}^{2}} \int_{0}^{\bar{x}_{2}} d x_{1}\left[-2 V_{1}^{\pi N}+3 V_{3}^{\pi N}+A_{3}^{\pi N}\right]\left(x_{i}\right)-\frac{2 x_{2} m_{N}^{2}}{q_{2}^{4}} \mathcal{V}_{1}^{\pi N, M(u)}+\frac{Q^{2}-q_{2}^{2}}{q_{2}^{4}} \widehat{V}_{123}^{\pi N}\right. \\
& \left.+\frac{Q^{2}+q_{2}^{2}}{q_{2}^{4}} \widehat{A}_{123}^{\pi N}-\frac{x_{2}^{2} m_{N}^{2}}{q_{2}^{4}}\left[\widehat{V}_{1345}^{\pi N}-2 \widehat{V}_{43}^{\pi N}+\widehat{A}_{34}^{\pi N}\right]-\frac{2 x_{2} m_{N}^{2}}{q_{2}^{4}} \widehat{\widehat{V}}_{123456}^{\pi N}\right\} \\
\mathcal{B}^{\pi N}= & -2 e_{d} \int_{0}^{1} d x_{3}\left\{\frac{1}{q_{3}^{2}} \int_{0}^{\bar{x}_{3}} d x_{1} V_{1}^{\pi N}\left(x_{i}\right)+\frac{m_{N}^{2}}{q_{3}^{4}} \mathcal{V}_{1}^{\pi N, M(d)}-\frac{x_{3} m_{N}^{2}}{q_{3}^{4}}\left[\widetilde{V}_{123}^{\pi N}-\widetilde{V}_{43}^{\pi N}\right]\right\} \\
& +2 e_{u} \int_{0}^{1} d x_{2}\left\{\frac{1}{q_{2}^{2}} \int_{0}^{\bar{x}_{2}} d x_{1}\left[V_{1}+A_{1}\right]^{\pi N}\left(x_{i}\right)+\frac{m_{N}^{2}}{q_{2}^{4}}\left[\mathcal{V}_{1}^{\pi N, M(u)}+\mathcal{A}_{1}^{\pi N, M(u)}\right]\right. \\
& \left.+\frac{x_{2} m_{N}^{2}}{q_{2}^{4}}\left[\widehat{V}_{1345}^{\pi N}+\widehat{V}_{123}^{\pi N}+\widehat{A}_{123}^{\pi N}-2 \widehat{V}_{43}^{\pi N}+\widehat{A}_{34}^{\pi N}\right]\right\}
\end{aligned}
$$

where we used shorthand notations for the combinations of the DAs:

$$
\begin{aligned}
& V_{43}=V_{4}-V_{3}, \\
& V_{123}=V_{1}-V_{2}-V_{3}, \\
& V_{1345}=-2 V_{1}+V_{3}+V_{4}+2 V_{5},
\end{aligned}
$$

$$
\begin{aligned}
& V_{12345}=2 V_{1}-V_{2}-V_{3}-V_{4}-V_{5}, \\
& V_{123456}=-V_{1}+V_{2}+V_{3}+V_{4}+V_{5}-V_{6},
\end{aligned}
$$

$A_{34}=A_{3}-A_{4}$,

$A_{123}=-A_{1}+A_{2}-A_{3}$, 


$$
\begin{aligned}
& A_{1345}=-2 A_{1}-A_{3}-A_{4}+2 A_{5}, \\
& A_{12345}=2 A_{1}-A_{2}+A_{3}+A_{4}-A_{5}, \\
& A_{123456}=A_{1}-A_{2}+A_{3}+A_{4}-A_{5}+A_{6}
\end{aligned}
$$

and also

$$
\begin{aligned}
& T_{137}=T_{1}-T_{3}-T_{7}, \\
& T_{13478}=2 T_{1}-T_{3}-T_{4}-T_{7}-T_{8}, \\
& T_{134678}=T_{1}-T_{3}-T_{4}+T_{6}-T_{7}-T_{8} .
\end{aligned}
$$

Explicit expressions for the functions $\mathcal{V}_{1}^{\pi N, M(u, d)}$ and $\mathcal{A}_{1}^{\pi N, M(u, d)}$ are given in Appendix B. The superscripts $(u)$ or $(d)$ stand for the cases that one of the $u$-quarks has the same position as the $d$-quark, and the two $u$-quarks have the same position, respectively. In addition, we use a compact notation

$$
q_{3} \equiv q-x_{3}(P+k), \quad q_{2} \equiv q-x_{2}(P+k)
$$

In the both expressions in (4.17) the functions with a "tilde" and a "hat" have $x_{3}$ and $x_{2}$ as an argument, respectively, cf. 4.15), 4.16). Also, in the terms involving two integrations over the momentum fractions, the remaining momentum fraction is replaced by using $x_{1}+x_{2}+x_{3}=1$.

The Borel transformation and the continuum subtraction are performed by using the following substitution rules:

$$
\begin{aligned}
& \int \mathrm{d} x \frac{\varrho(x)}{(q-x(P+k))^{2}}=-\int_{0}^{1} \frac{\mathrm{d} x}{x} \frac{\varrho(x)}{\left(s-P^{\prime 2}\right)} \rightarrow-\int_{x_{0}}^{1} \frac{\mathrm{d} x}{x} \varrho(x) \exp \left(-\frac{\bar{x} Q^{2}}{x M^{2}}-\frac{\bar{x} m_{N}^{2}}{M^{2}}\right) \\
& \int \mathrm{d} x \frac{\varrho(x)}{(q-x(P+k))^{4}}=\int_{0}^{1} \frac{\mathrm{d} x}{x^{2}} \frac{\varrho(x)}{\left(s-P^{\prime 2}\right)^{2}} \rightarrow \frac{1}{M^{2}} \int_{x_{0}}^{1} \frac{\mathrm{d} x}{x^{2}} \varrho(x) \exp \left(-\frac{\bar{x} Q^{2}}{x M^{2}}-\frac{\bar{x} m_{N}^{2}}{M^{2}}\right)+\frac{\varrho\left(x_{0}\right) e^{-s_{0} / M^{2}}}{Q^{2}+x_{0}^{2} m_{N}^{2}}
\end{aligned}
$$

where $M$ is the Borel parameter, $s=\frac{1-x}{x} Q^{2}+(1-x) m_{N}^{2}$ and $x_{0}$ is the solution of the corresponding quadratic equation for $s=s_{0}$ :

$$
x_{0}=\left[\sqrt{\left(Q^{2}+s_{0}-m_{N}^{2}\right)^{2}+4 m_{N}^{2} Q^{2}}-\left(Q^{2}+s_{0}-m_{N}^{2}\right)\right] /\left(2 m_{N}^{2}\right) .
$$

The contribution $\sim e^{-s_{0} / M^{2}}$ in Eq. (4.22) corresponds to the "surface terms" arising from the partial integration to reduce the power in the denominator $(q-x P)^{4}=\left(s-{P^{\prime}}^{2}\right)^{4}(-x)^{4}$ to the usual dispersion representation with the denominator $\sim$ $\left(s-{P^{\prime}}^{2}\right)$. Without continuum subtraction, i.e. in the limit $s_{0} \rightarrow \infty$ this term vanishes.

On the other hand, the nucleon contribution of interest corresponds to a pole term in the variable $P^{\prime 2}$ at $P^{\prime 2}=m_{N}^{2}$. For the relevant projections we get

$$
\begin{aligned}
\mathcal{A}^{\pi N} & =\frac{2 \lambda_{1}^{p}\left(Q^{2} / m_{N}^{2}\right) G_{1}^{\pi N}}{m_{N}^{2}-P^{\prime 2}}+\frac{2 \lambda_{\pi N} F_{1}\left(Q^{2}\right)}{m_{N}^{2}-\left(P^{\prime}-k\right)^{2}}, \\
\mathcal{B}^{\pi N} & =-\frac{\lambda_{1}^{p} G_{2}^{\pi N}}{m_{N}^{2}-P^{\prime 2}}+\frac{\lambda_{\pi N} F_{2}\left(Q^{2}\right)}{m_{N}^{2}-\left(P^{\prime}-k\right)^{2}}
\end{aligned}
$$

where $F_{1}$ and $F_{2}$ are the Dirac and Pauli proton (for $p \pi^{0}$ ) and neutron (for $n \pi^{+}$) electromagnetic form factors, respectively. The pion-nucleon coupling $\lambda_{\pi N} \equiv \lambda_{\pi N}\left(P^{\prime 2}\right)$ is defined in Eq. (4.9) and in general depends on the invariant mass $P^{\prime 2}$ of the pion-nucleon system. Close to threshold in the $P^{\prime 2}$ channel, $P_{\text {th }}^{\prime 2}=m_{N}^{2}(1+\delta)^{2}$, one obtains in the softpion limit $\lambda_{n \pi^{+}}=-\lambda_{1}^{p} / \sqrt{2}, \lambda_{p \pi^{0}}=-\lambda_{1}^{p} / 2$.

Note that the Borel transformation amounts in this case to the substitution

$$
\begin{aligned}
& \frac{1}{m_{N}^{2}-P^{\prime 2}} \rightarrow e^{-m_{N}^{2} / M^{2}}, \\
& \frac{1}{m_{N}^{2}-\left(P^{\prime}-k\right)^{2}} \rightarrow(1+\delta) e^{-\left[m_{N}^{2}(1+\delta)^{2}+\delta Q^{2}\right] / M^{2}} .
\end{aligned}
$$

One observes that the contribution of the pionnucleon intermediate state is suppressed compared to the nucleon contribution of interest by an extra factor $\exp \left\{\delta\left[m_{N}^{2}(2+\delta)+Q^{2}\right] / M^{2}\right\}$. Requiring that this suppression is at least as strong as $\exp \left\{-\left[s_{0}-m_{N}^{2}\right] / M^{2}\right\}$ in which case the pion-nucleon state has to be considered as a part of the continuum, we obtain

$$
\delta\left[m_{N}^{2}(2+\delta)+Q^{2}\right] \geq s_{0}-m_{N}^{2},
$$




$$
Q^{2} \geq\left(s_{0}-m_{N}^{2}\right) / \delta-(2+\delta) m_{N}^{2}
$$

where from $Q^{2} \geq 7.3 \mathrm{GeV}^{2}$. For smaller momentum transfers the pion-nucleon contribution has to be calculated explicitly and subtracted. The prob- lem is that the corresponding couplings $\lambda_{\pi N}$ have to be taken at the invariant mass of the pion-nucleon pair $P^{\prime 2}=m_{N}^{2}(1+\delta)^{2}+\delta Q^{2}$ and this dependence is not known a priory.

Neglecting the pion-nucleon intermediate state, we obtain the sum rules:

$$
\begin{aligned}
Q^{2} G_{1}^{\pi N}\left(Q^{2}\right) & =\frac{m_{N}^{2}}{2 \lambda_{1}^{p}}\left[\int_{x_{0}}^{1} \mathrm{~d} x\left(-\frac{\varrho_{2}^{a}(x)}{x}+\frac{\varrho_{4}^{a}(x)}{x^{2} M^{2}}\right) \exp \left(-\frac{\bar{x} Q^{2}}{x M^{2}}+\frac{x m_{N}^{2}}{M^{2}}\right)+\frac{\varrho_{4}^{a}\left(x_{0}\right) e^{-\left(s_{0}-m_{N}^{2}\right) / M^{2}}}{Q^{2}+x_{0}^{2} m_{N}^{2}}\right] \\
G_{2}^{\pi N}\left(Q^{2}\right) & =-\frac{1}{\lambda_{1}^{p}}\left[\int_{x_{0}}^{1} \mathrm{~d} x\left(-\frac{\varrho_{2}^{b}(x)}{x}+\frac{\varrho_{4}^{b}(x)}{x^{2} M^{2}}\right) \exp \left(-\frac{\bar{x} Q^{2}}{x M^{2}}+\frac{x m_{N}^{2}}{M^{2}}\right)+\frac{\varrho_{4}^{b}\left(x_{0}\right) e^{-\left(s_{0}-m_{N}^{2}\right) / M^{2}}}{Q^{2}+x_{0}^{2} m_{N}^{2}}\right]
\end{aligned}
$$

where the functions $\varrho_{2,4}^{a, b}(x)$ are given in terms of the generalized pion-nucleon distribution amplitudes as

$$
\begin{aligned}
\varrho_{2}^{a}(x)= & 2 e_{d}\left\{\widetilde{V}_{123}^{\pi N}+x \int_{0}^{\bar{x}} d x_{1} V_{3}^{\pi N}\left(x_{i}\right)\right\}+2 e_{u}\left\{x \int_{0}^{\bar{x}} d x_{1}\left[-2 V_{1}+3 V_{3}+A_{3}\right]^{\pi N}\left(x_{i}\right)-\widehat{V}_{123}^{\pi N}+\widehat{A}_{123}^{\pi N}\right\}, \\
\varrho_{4}^{a}(x)= & 2 e_{d}\left\{Q^{2} \widetilde{V}_{123}^{\pi N}+x^{2} m_{N}^{2} \widetilde{V}_{43}^{\pi N}\right\}+2 e_{u}\left\{Q^{2}\left(\widehat{V}_{123}^{\pi N}+\widehat{A}_{123}^{\pi N}\right)-x^{2} m_{N}^{2}\left[\widehat{V}_{1345}^{\pi N}-2 \widehat{V}_{43}^{\pi N}+\widehat{A}_{34}^{\pi N}\right]\right. \\
& \left.-2 x m_{N}^{2}\left(\mathcal{V}_{1}^{\pi N, M(u)}+\widehat{\widehat{V}}_{123456}^{\pi N}\right)\right\}, \\
\varrho_{2}^{b}(x)= & -2 e_{d}\left\{\int_{0}^{\bar{x}} d x_{1} V_{1}^{\pi N}\left(x_{i}\right)\right\}+2 e_{u}\left\{\int_{0}^{\bar{x}} d x_{1}\left[V_{1}+A_{1}\right]^{\pi N}\left(x_{i}\right)\right\} \\
\varrho_{4}^{b}(x)= & -2 e_{d} m_{N}^{2}\left\{\mathcal{V}_{1}^{\pi N, M(d)}-x\left[\widetilde{V}_{123}-\widetilde{V}_{43}\right]^{\pi N}\right\}+2 e_{u} m_{N}^{2}\left\{\left[\mathcal{V}_{1}^{\pi N, M(u)}+\mathcal{A}_{1}^{\pi N, M(u)}\right]\right. \\
& \left.+x\left[\widehat{V}_{1345}+\widehat{V}_{123}+\widehat{A}_{123}-2 \widehat{V}_{43}+\widehat{A}_{34}\right]^{\pi N}\right\} .
\end{aligned}
$$

Note that the sum rules have exactly the same structure as the sum rules for electromagnetic nucleon form factors in Ref. [34], with the only difference that nucleon DAs are replaced by the pion-nucleon ones.

For completeness, we quote also the sum rule for the axial form factor of the proton from Ref. 34] (for the charged current defined in (4.4) $)$ :

$$
G_{A}\left(Q^{2}\right)=\frac{1}{\lambda_{1}^{p}}\left[\int_{x_{0}}^{1} \mathrm{~d} x\left(-\frac{\varrho_{2}^{c}(x)}{x}+\frac{\varrho_{4}^{c}(x)}{x^{2} M^{2}}\right) \exp \left(-\frac{\bar{x} Q^{2}}{x M^{2}}+\frac{x m_{N}^{2}}{M^{2}}\right)+\frac{\varrho_{4}^{c}\left(x_{0}\right) e^{-\left(s_{0}-m_{N}^{2}\right) / M^{2}}}{Q^{2}+x_{0}^{2} m_{N}^{2}}\right]
$$

with

$$
\begin{aligned}
\varrho_{2}^{c}(x)= & \left\{\widetilde{V}_{123}+x \int_{0}^{\bar{x}} d x_{1} V_{3}\left(x_{i}\right)\right\}+\left\{x \int_{0}^{\bar{x}} d x_{1}\left[2 A_{1}+3 A_{3}+V_{3}\right]\left(x_{i}\right)-\widehat{A}_{123}+\widehat{V}_{123}\right\}, \\
\varrho_{4}^{c}(x)= & \left\{Q^{2} \widetilde{V}_{123}+x^{2} m_{N}^{2} \widetilde{V}_{43}\right\}+\left\{Q^{2}\left(\widehat{A}_{123}+\widehat{V}_{123}\right)+x^{2} m_{N}^{2}\left[\widehat{A}_{1345}-2 \widehat{A}_{34}+\widehat{V}_{43}\right]\right. \\
& \left.+2 x m_{N}^{2}\left(\mathcal{A}_{1}^{M(u)}-\widehat{\widehat{A}}_{123456}\right)\right\} .
\end{aligned}
$$

Note that in difference to Eq. (4.28) this sum rule involves nucleon DAs on the r.h.s., not the pion- 
nucleon ones.

\section{RESULTS}

The sum rules in Eqs. (4.28), (4.30) involve nucleon and pion-nucleon DA that can be expanded in contributions of conformal partial waves. To each order in the conformal expansion, one or several new nonperturbative parameters appear that can be related to matrix elements of local operators, as detailed in Ref. [40]. In the simplest approximation (asymptotic DAs) the sum rules depend on a single parameter which is the ratio of the twist- 4 and the twist-3 matrix elements of the three-quark operators of the lowest dimension (e.g. without derivatives) that differ by the quark spin projection on the lightcone. This ratio is known to ca. 20-30\% accuracy from the QCD sum rule calculations (see e.g. [34]). To the next-to-leading-order in the conformal spin, several more parameters enter that characterize the momentum fraction carried by a particular quark in the valence component of the nucleon wave function, both in the leading and higher twists. These parameters have been estimated using QCD sum rules [42, 43, 44] but the accuracy of such estimates is suspected to be low. By this reason we prefer to use the set of parameters that are intermediate between the asymptotic and the QCD sum rule motivated DAs, and are chosen in such a way that the nucleon electromagnetic and axial form factors are described well within the light-cone sum rule approach, see Ref. 34]. We will refer to this set of the DAs as the Braun-Lenz-Wittmann (BLW) model. For leading twist, these parameters are close to the model of Ref. [45].

In order to minimize further the uncertainty due to the choice of the DAs, the Borel parameter $M^{2}$ and the continuum threshold $s_{0}$, we present below the results for the ratios of the sum rules for the pion electroproduction amplitudes at threshold, Eqs. (4.28), to the nucleon axial form factor, Eq. (4.30):

$$
R_{1}^{\pi N}=\sqrt{2} Q^{2} G_{1}^{\pi N} /\left(G_{A} m_{N}^{2}\right)
$$

and

$$
R_{2}^{\pi N}=\sqrt{2} G_{2}^{\pi N} / G_{A} .
$$

For small momentum transfers, the first ratio is predicted to be one for the $\pi^{+} n$ and zero for $\pi^{0} n$ final state (4.12), while the second ratio vanishes in both cases in the soft-pion limit (4.13).

The LCSR (4.30) for the axial form factor itself is studied in the range $Q^{2}=1-10 \mathrm{GeV}^{2}$ in Ref. [34].
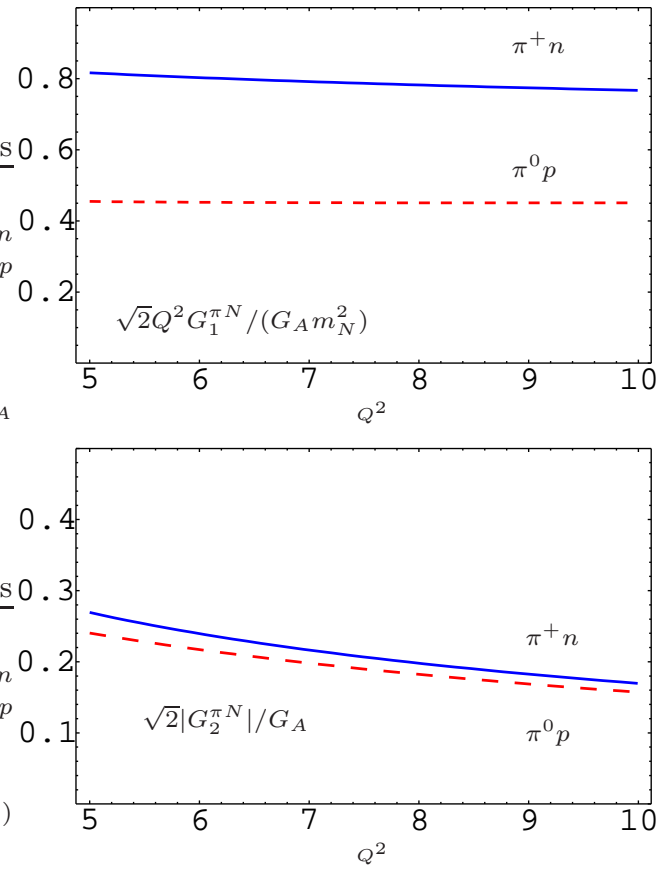

FIG. 3: Pion electroproduction amplitudes at threshold, (2.10) normalized to the nucleon axial form factor: $\pi^{+} n$ (solid blue curves) and $\pi^{0} p$ (dashed red curves). The calculation is done using the BLW model [34] for the nucleon DAs. The color identification refers to the online version.

The results are consistent (to $20 \%$ accuracy) with the dipole parametrization

$$
G_{A}\left(Q^{2}\right)=\frac{g_{A}}{\left(1+\frac{Q^{2}}{M_{A}^{2}}\right)^{2}},
$$

with $g_{A}=1.267 \pm 0.004$ and the mass parameter $M_{A}=1 \mathrm{GeV}$ that is suggested by both the neutrino scattering and pion electroproduction data at low $Q^{2}$.

The results are shown in Fig. 3f for the range of momentum transfers $Q^{2}=5-10 \mathrm{GeV}^{2}$. This range is chosen because for lower momenta the contribution of the pion-nucleon intermediate state becomes large and must be subtracted, and, on the other hand, for large momenta the (uncalculated) radiative corrections $\mathcal{O}\left(\alpha_{s}(M)\right)$ are expected to play an increasing role.

It attracts attention that the $R_{1}$ ratio is almost constant in the whole $Q^{2}$ range, i.e. the $Q^{2} G_{1}^{\pi N}$ amplitude turns out to be proportional to the axial form factor of the nucleon. This proportionality holds for all models of the nucleon DAs that we are considering, whereas the value of $R_{1}$ does depend on 
the shape of the DAs. We obtain

$$
\begin{gathered}
R_{1}^{\pi^{+} n}=0.80 \pm 0.10, \\
R_{1}^{\pi^{0} p}=0.45 \pm 0.08,
\end{gathered}
$$

where the given error estimate corresponds to the difference in the sum rule predictions using BLW and asymptotic DAs.

The second amplitude, $G_{2}^{\pi N}$, comes out similar in magnitude but different sign for the $\pi^{+} n$ and $\pi^{0} p$ final states. The sum rule results for the $R_{2}^{\pi N}$ ratio, shown in Fig. 3 can be parametrized as

$$
R_{2}^{\pi N}\left(Q^{2}\right)=\frac{R_{2}^{\pi N}\left(Q_{0}^{2}\right)}{1+b\left(Q^{2}-Q_{0}^{2}\right)}
$$

with the reference scale $Q_{0}^{2}=7.5 \mathrm{GeV}^{2}$, the slope $b \simeq 0.1 \mathrm{GeV}^{-2}$ and the values

$$
\begin{aligned}
& R_{2}^{\pi^{+} n}\left(Q_{0}^{2}\right)=0.21 \pm 0.15 \\
& R_{2}^{\pi^{0} p}\left(Q_{0}^{2}\right)=-0.19 \pm 0.07
\end{aligned}
$$

where the error estimates are obtained as in Eq. (5.4).

Using our central values for the amplitudes $G_{1,2}^{\pi N}$ we obtain the contribution to the structure function $F_{2}$ (2.11) which in the sum of all terms is almost the same as the corresponding contribution in the soft pion limit, cf. Eqs. (4.12), 4.13). This is in disagreement to Ref. 5] where a much larger structure function was obtained. The inspection shows that our result for the $\pi^{+} n$ production coincides with that of [5] within the errors, while our prediction for the $\pi^{0} p$ cross section is smaller by an order of magnitude. As the result, the DIS cross section obtained in [5] is completely dominated $(90 \%)$ by the contribution of the $\pi^{0} p$ state (compare Eqs. (11) and (12) in [5]), whereas in our calculation the $\pi^{0}$ to $\pi^{+}$production ratio is only of order $1 / 3$. Although the precise numbers are sensitive to the models of the nucleon DAs, there seems to be a qualitative difference that would be interesting to check experimentally.

\section{SUMMARY AND CONCLUSIONS}

We have given an analysis of the pion electroproduction amplitudes at threshold in the light-cone sum rule approach, using the methods of current algebra to construct the complete set of pion-nucleon distribution amplitudes in the soft-pion limit. The sum rule derived in this work are tree-level and may be affected by large (but calculable) radiative corrections. Having this in mind, the numerical results presented in Section 5 have to be considered as semiquantitative. Our calculation suggests that the $\pi^{0}$ to $\pi^{+}$production ratio becomes roughly of order $1 / 3$ at large momentum transfers $Q^{2}>7 \mathrm{GeV}^{2}$, and the amplitudes $G_{2}^{\pi^{0} p}$ and $G_{2}^{\pi^{+} n}$ are not small. They are predicted to be similar in magnitude and have opposite sign which can be tested in experiment using polarization transfer techniques. Working out the concrete experimental predictions (e.g. corresponding asymmetries) goes beyond the tasks of this paper and will be considered separately.

Main assumption underlying the present calculation is that the matching of the QCD operator expansion with the dispersion relation in terms of hadronic states can be done at sufficiently large scales where pions do not present independent degrees of freedom. In practice, the relevant values of the Borel parameter are close to the cutoff scale of the chiral perturbation theory, so this assumption may need a further check and better justification. Another direction of further work is obviously the inclusion of radiative corrections to the sum rules, which are enhanced at large momentum transfers. For practical applications one may need to add contributions of pole terms and generalize our analysis of the pion-nucleon distribution amplitudes to small off-threshold pion momenta by using the Omnes representation.

\section{Acknowledgements}

V.B. thanks M. V. Polyakov for the discussion that initiated our interest to this problem and useful comments. The work of D.I. was partially supported by grants RFBR-05-02-16211, NSh-5362.2006.2 and by the Helmholtz Association, grant VH-NG-004. The work by A.P. was supported by the Studienstiftung des deutschen Volkes. 


\section{APPENDICES}

\section{APPENDIX A: PION-NUCLEON GDAS OF HIGHER TWISTS}

In this Appendix we give without derivation the expressions for three-quark pion-nucleon generalized distribution amplitudes of higher twist.

\section{1. $n \pi^{+}$distribution amplitudes}

Definition of the GDAs follows Eq. (3.28). For the twist-4 we obtain:

$$
\begin{aligned}
V_{2}^{n \pi^{+}}(1,2,3)= & -\frac{1}{\sqrt{2}}\left\{\frac { 1 } { 2 } \left(\left\{A_{3}^{n}(1,3,2)-P_{1}^{n}(1,3,2)+S_{1}^{n}(1,3,2)-T_{3}^{n}(1,3,2)\right.\right.\right. \\
& \left.\left.\left.-T_{7}^{n}(1,3,2)-V_{3}^{n}(1,3,2)\right\}+\{1 \leftrightarrow 2\}\right)\right\}, \\
A_{2}^{n \pi^{+}}(1,2,3)= & -\frac{1}{\sqrt{2}}\left\{\frac { 1 } { 2 } \left(\left\{A_{3}^{n}(1,3,2)+P_{1}^{n}(1,3,2)-S_{1}^{n}(1,3,2)+T_{3}^{n}(1,3,2)\right.\right.\right. \\
& \left.\left.\left.+T_{7}^{n}(1,3,2)-V_{3}^{n}(1,3,2)\right\}-\{1 \leftrightarrow 2\}\right)\right\}, \\
V_{3}^{n \pi^{+}}(1,2,3)= & \frac{1}{\sqrt{2}}\left\{\frac { 1 } { 2 } \left(\left\{A_{2}^{n}(1,3,2)-P_{1}^{n}(1,3,2)+S_{1}^{n}(1,3,2)+T_{3}^{n}(1,3,2)\right.\right.\right. \\
& \left.\left.\left.+T_{7}^{n}(1,3,2)+V_{2}^{n}(1,3,2)\right\}+\{1 \leftrightarrow 2\}\right)\right\}, \\
& \left.\left.\left.-V_{3}^{n}(1,3,2)\right\}-\{1 \leftrightarrow 2\}\right)\right\}, \\
& \left.\left.\left.+V_{3}^{n}(1,3,2)\right\}-\{1 \leftrightarrow 2\}\right)\right\}, \\
P_{1}^{n \pi^{+}}(1,2,3)= & -\frac{1}{\sqrt{2}}\left\{\frac { 1 } { 4 } \left(\left\{-A_{2}^{n}(1,3,2)-A_{3}^{n}(1,3,2)+P_{1}^{n}(1,3,2)+S_{1}^{n}(1,3,2)\right.\right.\right. \\
& \left.\left.\left.+T_{7}^{n}(1,3,2)-V_{2}^{n}(1,3,2)\right\}-\{1 \leftrightarrow 2\}\right)\right\} \\
& \frac{1}{\sqrt{2}}\left\{\frac { 1 } { 2 } \left(\left\{-A_{2}^{n}(1,3,2)-P_{1}^{n}(1,3,2)+S_{1}^{n}(1,3,2)+T_{3}^{n}(1,3,2)\right.\right.\right. \\
S_{1}^{n \pi^{+}}(1,2,3)= & \frac{1}{\sqrt{2}}\left\{\frac { 1 } { 4 } \left(\left\{A_{2}^{n}(1,3,2)+A_{3}^{n}(1,3,2)+P_{1}^{n}(1,3,2)+S_{1}^{n}(1,3,2)\right.\right.\right. \\
& +T_{3}^{n}(1,3,2)-T_{7}^{n}(1,3,2)-V_{2}^{n}(1,3,2) \\
& \\
&
\end{aligned}
$$




$$
\begin{aligned}
T_{2}^{n \pi^{+}}(1,2,3)= & -\frac{1}{\sqrt{2}}\left\{\frac { 1 } { 2 } \left(\left\{P_{1}^{n}(1,3,2)+S_{1}^{n}(1,3,2)-T_{3}^{n}(1,3,2)+T_{7}^{n}(1,3,2)\right\}\right.\right. \\
& +\{1 \leftrightarrow 2\})\}, \\
T_{3}^{n \pi^{+}}(1,2,3)= & \frac{1}{\sqrt{2}}\left\{\frac { 1 } { 4 } \left(\left\{A_{2}^{n}(1,3,2)-A_{3}^{n}(1,3,2)-P_{1}^{n}(1,3,2)-S_{1}^{n}(1,3,2)\right.\right.\right. \\
& +2 T_{2}^{n}(1,3,2)-T_{3}^{n}(1,3,2)+T_{7}^{n}(1,3,2)-V_{2}^{n}(1,3,2) \\
& \left.\left.\left.-V_{3}^{n}(1,3,2)\right\}+\{1 \leftrightarrow 2\}\right)\right\}, \\
T_{7}^{n \pi^{+}}(1,2,3)= & \frac{1}{\sqrt{2}}\left\{\frac { 1 } { 4 } \left(\left\{A_{2}^{n}(1,3,2)-A_{3}^{n}(1,3,2)+P_{1}^{n}(1,3,2)+S_{1}^{n}(1,3,2)\right.\right.\right. \\
& -2 T_{2}^{n}(1,3,2)+T_{3}^{n}(1,3,2)-T_{7}^{n}(1,3,2)-V_{2}^{n}(1,3,2) \\
& \left.\left.\left.-V_{3}^{n}(1,3,2)\right\}+\{1 \leftrightarrow 2\}\right)\right\} .
\end{aligned}
$$

The expressions for twist-5 GDAs are identical up to the substitution

$$
\begin{aligned}
\left\{S_{1}^{n \pi^{+}}, P_{1}^{n \pi^{+}}, V_{2}^{n \pi^{+}},\right. & \left.V_{3}^{n \pi^{+}}, A_{2}^{n \pi^{+}}, A_{3}^{n \pi^{+}}, T_{2}^{n \pi^{+}}, T_{3}^{n \pi^{+}}, T_{7}^{n \pi^{+}}\right\} \rightarrow \\
& \rightarrow\left\{S_{2}^{n \pi^{+}}, P_{2}^{n \pi^{+}}, V_{5}^{n \pi^{+}}, V_{4}^{n \pi^{+}}, A_{5}^{n \pi^{+}}, A_{4}^{n \pi^{+}}, T_{5}^{n \pi^{+}}, T_{4}^{n \pi^{+}}, T_{8}^{n \pi^{+}}\right\},
\end{aligned}
$$

and the similar replacement of the neutron DAs on the r.h.s.

Finally, the expressions for twist-6 GDAs are identical to twist-3 with the substitution

$$
\left\{V_{1}^{n \pi^{+}}, A_{1}^{n \pi^{+}}, T_{1}^{n \pi^{+}}\right\} \rightarrow\left\{V_{6}^{n \pi^{+}}, A_{6}^{n \pi^{+}}, T_{6}^{n \pi^{+}}\right\}
$$

and the similar substitution of the neutron DAs.

\section{2. $p \pi^{0}$ distribution amplitudes}

Definition of the GDAs follows Eq. (3.28). For twist-4 we obtain

$$
\begin{aligned}
& S_{1}^{p \pi^{0}}(1,2,3)=-\frac{1}{2}\left\{2 P_{1}^{p}(1,3,2)-S_{1}^{p}(1,2,3)\right\}, \\
& P_{1}^{p \pi^{0}}(1,2,3)=-\frac{1}{2}\left\{2 S_{1}^{p}(1,3,2)-P_{1}^{p}(1,2,3)\right\}, \\
& V_{2}^{p \pi^{0}}(1,2,3)=\frac{1}{2} V_{2}^{p}(1,2,3), \quad V_{3}^{p \pi^{0}}(1,2,3)=\frac{1}{2} V_{3}^{p}(1,2,3), \\
& A_{2}^{p \pi^{0}}(1,2,3)=\frac{1}{2} A_{2}^{p}(1,2,3), \quad A_{3}^{p \pi^{0}}(1,2,3)=\frac{1}{2} A_{3}^{p}(1,2,3), \\
& T_{2}^{p \pi^{0}}(1,2,3)=-\frac{1}{2} T_{2}^{p}(1,2,3), \quad T_{3}^{p \pi^{0}}(1,2,3)=\frac{1}{2}\left\{T_{3}^{p}(1,2,3)+2 T_{7}^{p}(1,2,3)\right\}, \\
& T_{7}^{p \pi^{0}}(1,2,3)=\frac{1}{2}\left\{T_{7}^{p}(1,2,3)+2 T_{3}^{p}(1,2,3)\right\} .
\end{aligned}
$$

The expressions for twist-5 and twist-6 GDAs are identical to those for twist-4 and twist-3, respectively, with obvious substitutions as described for the $n \pi^{+}$case. 


\section{3. $p \pi^{+}$distribution amplitudes}

For completeness we present here the expressions for the $p \pi^{+}$GDAs. They are defined as the corresponding matrix element of the trilocal light-ray operator

$$
\hat{O}_{\alpha \gamma \beta}^{u u u}=\epsilon^{i j k} u_{\alpha}^{i}\left(a_{1} z\right) u_{\beta}^{j}\left(a_{2} z\right) u_{\gamma}^{k}\left(a_{3} z\right)
$$

and can be relevant, e.g. for weak form factors corresponding to the charged $W$-boson exchange. Working out the necessary commutators we obtain

$$
\begin{aligned}
& {\left[Q_{5}^{1}, \hat{O}_{\alpha \beta \gamma}^{u u u}(z)\right]=-\frac{1}{2}\left\{\left(\gamma_{5}\right)_{\alpha \lambda} \hat{O}_{\lambda \beta \gamma}^{\text {duu }}(z)+\left(\gamma_{5}\right)_{\beta \lambda} \hat{O}_{\alpha \lambda \gamma}^{u d u}(z)+\left(\gamma_{5}\right)_{\gamma \lambda} \hat{O}_{\alpha \beta \lambda}^{u u d}(z)\right\},} \\
& {\left[Q_{5}^{2}, \hat{O}_{\alpha \beta \gamma}^{u u u}(z)\right]=\frac{i}{2}\left\{\left(\gamma_{5}\right)_{\alpha \lambda} \hat{O}_{\lambda \beta \gamma}^{d u u}(z)+\left(\gamma_{5}\right)_{\beta \lambda} \hat{O}_{\alpha \lambda \gamma}^{u d u}(z)+\left(\gamma_{5}\right)_{\gamma \lambda} \hat{O}_{\alpha \beta \lambda}^{u u d}(z)\right\},}
\end{aligned}
$$

In fact, a separate calculation is not needed in this case. For all GDAs:

$$
\begin{aligned}
& S_{i}^{p \pi^{+}}(1,2,3)=-S_{i}^{n \pi^{+}}(1,2,3)+S_{i}^{p}(1,2,3), \\
& P_{i}^{p \pi^{+}}(1,2,3)=-P_{i}^{n \pi^{+}}(1,2,3)+P_{i}^{p}(1,2,3), \\
& V_{i}^{p \pi^{+}}(1,2,3)=V_{i}^{n \pi^{+}}(1,2,3)+V_{i}^{p}(1,2,3), \\
& A_{i}^{p \pi^{+}}(1,2,3)=A_{i}^{n \pi^{+}}(1,2,3)+A_{i}^{p}(1,2,3), \\
& T_{i}^{p \pi^{+}}(1,2,3)=-T_{i}^{n \pi^{+}}(1,2,3)+T_{i}^{p}(1,2,3) .
\end{aligned}
$$

\section{APPENDIX B: $x^{2}$-CORRECTIONS}

\section{1. $\mathcal{V}_{1}^{\pi N, M}$ corrections}

The method to calculate the $\mathcal{O}\left(x^{2}\right)$ contributions in the OPE of the baryon operators in a diquark-quark configuration (i.e. when the light-cone positions of two quarks coincide) is described in detail in Refs. 30, 34] and need not be repeated here. The functions $\mathcal{V}^{M}$ can be found as solutions of the following equations for the moments:

$$
\begin{aligned}
\int \mathrm{d} x_{3} x_{3}^{n} \mathcal{V}_{1}^{\pi N, M(d)}\left(x_{3}\right)= & \frac{1}{(n+1)(n+2)}\left[\left(-2 V_{1}+V_{3}+V_{4}+2 V_{5}\right)^{(d)(n+2)}\right]^{\pi N} \\
& +\frac{1}{(n+1)(n+3)}\left[(n+3) V_{1}^{(d)(n+2)}+\left(V_{1}-V_{2}\right)^{(d)(n+2)}-\left(V_{1}+V_{5}\right)(d)(n+1)\right]^{\pi N}, \\
\int \mathrm{d} x_{2} x_{2}^{n} \mathcal{V}_{1}^{\pi N, M(u)}\left(x_{2}\right)= & \frac{1}{(n+1)(n+3)}\left[(n+3) V_{1}^{(u)(n+2)}+\left(V_{1}-V_{2}\right)^{(u)(n+2)}\right]^{\pi N} \\
& +\frac{1}{(n+1)(n+2)}\left[\left(-2 V_{1}+V_{3}+V_{4}+2 V_{5}\right)^{(u)(n+2)}\right]^{\pi N} .
\end{aligned}
$$

We present the solutions as

$$
\begin{aligned}
& \mathcal{V}_{1}^{\pi N, M(u)}=\frac{1}{24}\left\{\lambda_{1} C_{\lambda}^{u, \pi N}+f_{N} C_{f}^{u, \pi N}\right\} \\
& \mathcal{V}_{1}^{\pi N, M(d)}=\frac{1}{24}\left\{\lambda_{1} C_{\lambda}^{d, \pi N}+f_{N} C_{f}^{d, \pi N}\right\}
\end{aligned}
$$


where

$$
\begin{aligned}
& C_{\lambda}^{u, p \pi^{0}}=-\frac{1}{2}\left(1-x_{2}\right)^{3}\left\{6+18 x_{2}+19 x_{2}^{2}+10 f_{1}^{u} x_{2}^{2}-3\left(9+10 f_{1}^{u}\right) x_{2}^{3}-4 f_{1}^{d}\left(1+3 x_{2}+26 x_{2}^{2}-25 x_{2}^{3}\right)\right\}, \\
& C_{f}^{u, p \pi^{0}}=\frac{1}{2}\left(1-x_{2}\right)^{3}\left\{6+18 x_{2}+143 x_{2}^{2}+297 x_{2}^{3}-408 x_{2}^{4}-10 A_{1}^{u}\left(1-3 x_{2}\right) x_{2}^{2}\right. \\
& \left.-2 V_{1}^{d}\left(4+12 x_{2}+7 x_{2}^{2}+599 x_{2}^{3}-612 x_{2}^{4}\right)\right\} \\
& C_{\lambda}^{d, p \pi^{0}}=-\frac{1}{2}\left\{3 x_{3}^{2}\left(1-x_{3}\right)\left(87-33 x_{3}-13 x_{3}^{2}+11 x_{3}^{3}\right)+156 x_{3}^{2} \ln \left[x_{3}\right]\right. \\
& \left.+2 f_{1}^{d}\left[\left(1-x_{3}\right)\left(4+4 x_{3}-351 x_{3}^{2}+169 x_{3}^{3}+49 x_{3}^{4}-55 x_{3}^{5}\right)-180 x_{3}^{2} \ln \left[x_{3}\right]\right]\right\}, \\
& C_{f}^{d, p \pi^{0}}=-\frac{1}{2}\left\{x_{3}^{2}\left(1-x_{3}\right)\left(1979-877 x_{3}-1513 x_{3}^{2}+2232 x_{3}^{3}-816 x_{3}^{4}\right)+996 x_{3}^{2} \ln \left[x_{3}\right]\right. \\
& \left.-4 V_{1}^{d}\left[\left(1-x_{3}\right)\left(4+4 x_{3}+1527 x_{3}^{2}-893 x_{3}^{3}-923 x_{3}^{4}+1613 x_{3}^{5}-612 x_{3}^{6}\right)+720 x_{3}^{2} \ln \left[x_{3}\right]\right]\right\}, \\
& C_{\lambda}^{u, n \pi^{+}}=-\frac{1}{\sqrt{2}}\left(1-x_{2}\right)^{3}\left\{2+6 x_{2}-75 x_{2}^{2}+43 x_{2}^{3}+4 f_{1}^{u}\left(1+3 x_{2}+26 x_{2}^{2}-25 x_{2}^{3}\right)\right. \\
& \left.+2 f_{1}^{d}\left(4+12 x_{2}+89 x_{2}^{2}-55 x_{2}^{3}\right)\right\} \\
& C_{f}^{u, n \pi^{+}}=-\frac{1}{\sqrt{2}}\left(1-x_{2}\right)^{3}\left\{10+30 x_{2}+425 x_{2}^{2}-337 x_{2}^{3}-30 V_{1}^{d}\left(1-3 x_{2}\right) x_{2}^{2}\right. \\
& \left.-2 A_{1}^{u}\left(4+12 x_{2}+17 x_{2}^{2}+569 x_{2}^{3}-612 x_{2}^{4}\right)\right\} \\
& C_{\lambda}^{d, n \pi^{+}}=\frac{1}{\sqrt{2}}\left\{\left(1-x_{3}\right)\left(-8-8 x_{3}+441 x_{3}^{2}-239 x_{3}^{3}-59 x_{3}^{4}+77 x_{3}^{5}\right)+204 x_{3}^{2} \ln \left[x_{3}\right]\right. \\
& \left.+2\left(f_{1}^{u}+2 f_{1}^{d}\right)\left[\left(1-x_{3}\right)\left(4+4 x_{3}-351 x_{3}^{2}+169 x_{3}^{3}+49 x_{3}^{4}-55 x_{3}^{5}\right)-180 x_{3}^{2} \ln \left[x_{3}\right]\right]\right\}, \\
& C_{f}^{d, n \pi^{+}}=-\frac{1}{\sqrt{2}}\left\{\left(1-x_{3}\right)\left(16+16 x_{3}+171 x_{3}^{2}-941 x_{3}^{3}+847 x_{3}^{4}-217 x_{3}^{5}\right)-108 x_{3}^{2} \ln \left[x_{3}\right]\right. \\
& \left.+4 A_{1}^{u}\left[\left(1-x_{3}\right)\left(4+4 x_{3}+1527 x_{3}^{2}-893 x_{3}^{3}-923 x_{3}^{4}+1613 x_{3}^{5}-612 x_{3}^{6}\right)+720 x_{3}^{2} \ln \left[x_{3}\right]\right]\right\} .
\end{aligned}
$$

\section{2. $\mathcal{A}_{1}^{\pi N, M}$ corrections}

Similarly we get the equations for the $\mathcal{A}^{M}$ functions:

$$
\begin{aligned}
\int \mathrm{d} x_{3} x_{3}^{n} \mathcal{A}_{1}^{\pi N, M(d)}\left(x_{3}\right)= & \frac{1}{(n+1)(n+2)}\left[\left(-2 A_{1}+A_{3}+A_{4}+2 A_{5}\right)^{(d)(n+2)}\right]^{\pi N} \\
& +\frac{1}{(n+1)(n+3)}\left[(n+3) A_{1}^{(d)(n+2)}+\left(A_{1}-A_{2}\right)^{(d)(n+2)}-\left(A_{1}+A_{5}\right)^{(d)(n+1)}\right]^{\pi N}, \\
\int \mathrm{d} x_{2} x_{2}^{n} \mathcal{A}_{1}^{\pi N, M(u)}\left(x_{2}\right)= & \frac{1}{(n+1)(n+3)}\left[(n+3) A_{1}^{(u)(n+2)}+\left(A_{1}-A_{2}\right)^{(u)(n+2)}\right]^{\pi N} \\
& +\frac{1}{(n+1)(n+2)}\left[\left(-2 A_{1}+A_{3}+A_{4}+2 A_{5}\right)^{(u)(n+2)}\right]^{\pi N},
\end{aligned}
$$


We obtain

$$
\begin{aligned}
& \mathcal{A}_{1}^{\pi N, M(d)}=0 \\
& \mathcal{A}_{1}^{\pi N, M(u)}=\frac{1}{24}\left(1-x_{2}\right)^{3}\left\{\lambda_{1} D_{\lambda}^{u, \pi N}+f_{N} D_{f}^{u, \pi N}\right\} .
\end{aligned}
$$

with the coefficients

$$
\begin{aligned}
D_{\lambda}^{u, p \pi^{0}}= & -\frac{1}{2}\left\{x_{2}^{2}\left(29-45 x_{2}\right)+2 f_{1}^{u}\left(4+12 x_{2}-51 x_{2}^{2}+45 x_{2}^{3}\right)-4 f_{1}^{d}\left(2+6 x_{2}+17 x_{2}^{2}-30 x_{2}^{3}\right)\right\}, \\
D_{f}^{u, p \pi^{0}}= & -\frac{1}{2}\left\{8+24 x_{2}-21 x_{2}^{2}+2 A_{1}^{u}\left(4+12 x_{2}-9 x_{2}^{2}-609 x_{2}^{3}+612 x_{2}^{4}\right)+45 x_{2}^{3}+10 V_{1}^{d} x_{2}^{2}\left(1-3 x_{2}\right)\right\}, \\
D_{\lambda}^{u, n \pi^{+}}= & \frac{1}{\sqrt{2}}\left\{3 x_{2}^{2}\left(47-55 x_{2}\right)-4 f_{1}^{u}\left(2+6 x_{2}+17 x_{2}^{2}-30 x_{2}^{3}\right)+2 f_{1}^{d}\left(4+12 x_{2}-221 x_{2}^{2}+255 x_{2}^{3}\right)\right\}, \\
D_{f}^{u, n \pi^{+}}= & \frac{1}{\sqrt{2}}\left\{16+48 x_{2}-35 x_{2}^{2}+1323 x_{2}^{3}-1224 x_{2}^{4}+2 A_{1}^{u}\left(8+24 x_{2}-13 x_{2}^{2}-1233 x_{2}^{3}+1224 x_{2}^{4}\right)\right. \\
& \left.+6 V_{1}^{d}\left(4+12 x_{2}-9 x_{2}^{2}-609 x_{2}^{3}+612 x_{2}^{4}\right)\right\} .
\end{aligned}
$$

[1] N. M. Kroll, M. A. Ruderman Phys. Rev. 93, 233 (1954).

[2] Y. Nambu and E. Shrauner, Phys. Rev. 128, 862 (1962).

[3] A. I. Vainshtein and V. I. Zakharov, Nucl. Phys. B 36, 589 (1972).

[4] S. Scherer and J. H. Koch, Nucl. Phys. A 534, 461 (1991).

[5] P. V. Pobylitsa, M. V. Polyakov and M. Strikman, Phys. Rev. Lett. 87 (2001) 022001.

[6] V. L. Chernyak and A. R. Zhitnitsky, JETP Lett. 25, 510 (1977) Sov. J. Nucl. Phys. 31, 544 (1980); V. L. Chernyak, A. R. Zhitnitsky and V. G. Serbo, JETP Lett. 26, 594 (1977) Sov. J. Nucl. Phys. 31, 552 (1980).

[7] A. V. Radyushkin, JINR report R2-10717 (1977), arXiv:hep-ph/0410276 (English translation);

A. V. Efremov and A. V. Radyushkin, Theor. Math. Phys. 42, 97 (1980) Phys. Lett. B 94, 245 (1980).

[8] G. P. Lepage and S. J. Brodsky, Phys. Lett. B 87, 359 (1979); Phys. Rev. D 22, 2157 (1980).

[9] B. Pire and L. Szymanowski, Phys. Lett. B 622, 83 (2005).

[10] J. P. Lansberg, B. Pire and L. Szymanowski, arXiv:hep-ph/0610407

[11] N. Isgur and C. H. Llewellyn Smith, Phys. Rev. Lett. 52, 1080 (1984).

[12] N. Isgur and C. H. Llewellyn Smith, Nucl. Phys. B 317, 526 (1989), Phys. Lett. B 217 (1989) 535.

[13] P. Kroll, M. Schurmann and P. A. M. Guichon, Nucl. Phys. A 598, 435 (1996).

[14] A. V. Radyushkin, Phys. Rev. D 58, 114008 (1998).
[15] M. Diehl, T. Feldmann, R. Jakob and P. Kroll, Eur. Phys. J. C 8, 409 (1999).

[16] K. Goeke, M. V. Polyakov and M. Vanderhaeghen, Prog. Part. Nucl. Phys. 47, 401 (2001).

[17] M. Diehl, Phys. Rept. 388, 41 (2003).

[18] A. V. Belitsky and A. V. Radyushkin, Phys. Rept. 418, 1 (2005).

[19] A. V. Belitsky, X. d. Ji and F. Yuan, Phys. Rev. D 69, 074014 (2004).

[20] M. Diehl, T. Feldmann, R. Jakob and P. Kroll, Eur. Phys. J. C 39, 1 (2005).

[21] M. Guidal, M. V. Polyakov, A. V. Radyushkin and M. Vanderhaeghen, Phys. Rev. D 72, 054013 (2005).

[22] P. A. M. Guichon, L. Mosse and M. Vanderhaeghen, Phys. Rev. D 68, 034018 (2003).

[23] M. V. Polyakov and S. Stratmann, arXiv:hep-ph/0609045.

[24] I. I. Balitsky, V. M. Braun and A. V. Kolesnichenko, Nucl. Phys. B 312, 509 (1989).

[25] V. L. Chernyak and I. R. Zhitnitsky, Nucl. Phys. B 345, 137 (1990).

[26] V. M. Braun, A. Khodjamirian and M. Maul, Phys. Rev. D 61, 073004 (2000).

[27] J. Bijnens and A. Khodjamirian, Eur. Phys. J. C 26, 67 (2002).

[28] V. M. Braun, arXiv:hep-ph/9801222

[29] P. Colangelo and A. Khodjamirian, arXiv:hep-ph/0010175.

[30] V. M. Braun, A. Lenz, N. Mahnke and E. Stein, Phys. Rev. D 65, 074011 (2002).

[31] Z. G. Wang, S. L. Wan and W. M. Yang, Phys. Rev. D 73, 094011 (2006). 
[32] Z. G. Wang, S. L. Wan and W. M. Yang, Eur. Phys. J. C 47, 375 (2006).

[33] A. Lenz, M. Wittmann and E. Stein, Phys. Lett. B 581, 199 (2004).

[34] V. M. Braun, A. Lenz and M. Wittmann, Phys. Rev. D 73, 094019 (2006).

[35] M. Q. Huang and D. W. Wang, Phys. Rev. D 69, 094003 (2004).

[36] M. Q. Huang and D. W. Wang, arXiv:hep-ph/0608170

[37] Z. G. Wang, arXiv:hep-ph/0609155.

[38] V. M. Braun, A. Lenz, G. Peters and A.V. Radyushkin, Phys. Rev. D 73 (2006) 034020.

[39] J. D. Bjorken and S. D. Drell, Relativistic Quantum
Fields (McGraw-Hill, New York, 1965).

[40] V. Braun, R. J. Fries, N. Mahnke and E. Stein, Nucl. Phys. B 589 (2000) 381 [Erratum-ibid. B 607 (2001) 433].

[41] B. L. Ioffe, Nucl. Phys. B 188, 317 (1981) [Erratumibid. B 191, 591 (1981)].

[42] V. L. Chernyak and I. R. Zhitnitsky, Nucl. Phys. B 246, 52 (1984).

[43] I. D. King and C. T. Sachrajda, Nucl. Phys. B 279, 785 (1987).

[44] V. L. Chernyak, A. A. Ogloblin and I. R. Zhitnitsky, Z. Phys. C 42, 569 (1989).

[45] J. Bolz and P. Kroll, Z. Phys. A 356 (1996) 327. 